International Journal of Pure and Applied Mathematics

Volume 95 No. 3 2014, 463-491

ISSN: 1311-8080 (printed version); ISSN: 1314-3395 (on-line version)

url: http://www.ijpam.eu

doi: http://dx.doi.org/10.12732/ijpam.v95i3.12

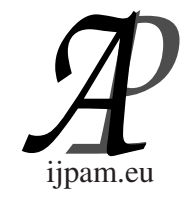

\title{
MODELING THE POWER ALLOCATION IN DYNAMIC SPECTRUM ACCESS FOR COGNITIVE RADIOS AS A GENERALIZED NASH EQUILIBRIUM PROBLEM
}

\author{
Laura Prati \\ Department of Information Engineering \\ University of Florence \\ 3, Via S. Marta, I-50139, Florence, ITALY
}

\begin{abstract}
The demand of radio resources like mobile phones and Internet services is dramatically increasing and inefficiency in the traditional allocation of electromagnetic spectrum is apparent. A novel paradigm for allocating resources is the Dynamic Spectrum Access, where primary and secondary users share the same frequency band.

In this paper, in a Cognitive Radio context, we model the power allocation for secondary users coexisting in the same frequency band with primary ones as a Generalized Nash Equilibrium Problem. We develop a theoretic analysis showing existence and uniqueness of the Generalized Nash Equilibrium and convergence of the Best Response Algorithm to it. In practical implementations, the conditions we propose are natural (a lower and an upper bound for the Signal-to-Interference-plus-Noise-Ratio of the radio devices are involved) and easy to be locally verified by users, which just need the knowledge of the total interference they experience near their own receiver.
\end{abstract}

AMS Subject Classification: 91A28, 91A40, 91A80

Key Words: generalized Nash equilibrium problem, cognitive radio, power allocation, game theory

\section{Introduction}

The radio spectrum is a finite resource managed by State governments. Complex na-

Received: July 2, 2014

(c) 2014 Academic Publications, Ltd. url: www.acadpubl.eu 
tional and international rules govern its occupancy and use. This regulation includes the a priori assignment of frequency bands, the specification of maximum allowed power levels and of the maximum allowed interference with other potential users [1].

Because of the rapidly increasing demand of radio resources (mobile phones, Internet services, etc.), such a traditional method for the allocation of the frequency band to a given communication system is becoming inefficient. Engaged frequency bands coexist with huge under-utilized bands dedicated to different services and users [1], [2], [3], [4], [5] and [6]. In particular, currently the licensed part of the spectrum is the one poorly utilized [2], [6] and [7].

Consequently, it has become increasingly important to develop new methods in order to utilize the electromagnetic spectrum more efficiently and to solve problems of radio-band congestion. Innovative artificial techniques in radio science and new paradigms in radio communication protocols have been suggested in order to improve the use of fixed bands [8] and [9]. But another approach could be employed. Signals could be transmitted not on a formerly fixed frequency band, but, more dynamically, on a frequency band available in specific time and location, a so called spectrum hole [10].

A system able to adapt to environmental conditions would be therefore useful to improve the use of the spectrum. A Cognitive Radio (CR) system is therefore promising in order to obtain more efficiency, basing on flexibility and dynamism [11], [12] and [13]. Indeed, a Cognitive Radio is a communication system able to sense the operating environment and to adapt in order to obtain some purposes, by respecting the constraints imposed by the environment itself [14], [15], [16] and [17].

A Cognitive Radio approach to spectrum sharing is not limited to the unlicensed bands, but it is also extended to the licensed ones, leading to a coexistence problem of different users, all willing to independently reach a good quality signal, in spite of the presence of the other users. In such a Dynamic Spectrum Access (DSA), primary users are the owners of the spectrum rights in a specific frequency band, and the secondary users are expected to share the same band without causing harmful interference to the primary ones; for this purpose, primary users select a cap on the total interference they are going to tolerate from the secondary ones [11].

In a Cognitive radio context, Game Theory accounts the radio devices as players aiming to optimize their own service quality by means of the choice of their own transmitting power. Indeed, transmitting at the maximum power does not imply the best choice in order to obtain a good signal at a radio receiver. The high interference created by a user induces all the other users to rise their transmitting powers: as a result, the mentioned user indirectly determines an unwanted interference, eventually obtaining a bad quality signal and other practical issues such as a faster consumption for the battery (for the specific tool of saving as much battery energy as possible see 
[18] and references therein). Moreover, the distributed approach of Game Theory is suitable: decisions are not taken in a centralized way. Players regulate their own transmitting power according to the knowledge of locally available data (such as the total received interference).

Several models have been proposed in the literature to describe similar competing situations and to establish the behavior that users in the same band should adopt to reach a satisfying service quality, see [19] for an overview. A Game theoretic approach to the power control problem in wireless systems has been offered in [20], [21], [22], [23] and [24], where service preferences for each user are represented by utility functions to be maximized. In a major work, [25], a model for power control where the utility functions reflect the level of satisfaction that users get from using system resources is considered. A linear pricing to the net utility function is introduced in order to improve the model and mathematical conditions are given to guarantee the existence (not the uniqueness) of a Nash Equilibrium for the Supermodular Game. In [26] a graphical method is used to prove existence and uniqueness of the Nash Equilibrium in a power control game in which the selfish behavior of the secondary users is mitigated thanks to the specific choice of pricing. In [27] a discrete-time model, originating from the net utility function of [25] and pricing in [26], is proposed. The presence of a primary user is included. This Game shows satisfying performance in terms of employed powers, nevertheless the choice of developing a time-discrete model, where instant after instant the knowledge of a specific term of pricing is obtained, let the authors fall into a linear case studied in [25] which gives conditions ensuring the existence, but not the uniqueness, of a Nash Equilibrium.

In a previous work, see [28], we have modeled a Generalized Nash Equilibrium Problem for Centralized Dynamic Spectrum Leasing (C-DSL, where also primary users, not only the secondary ones, are active players of the game), following [27] for the secondary utility, but with the introduction of multiple primary users and overall assuming that the secondary utility of player $i$ depends on simultaneous choices of players (like in [25]) instead of on their previous choices.

In the present paper, we focus again on the secondary utility function of [28] but in a Dynamic Spectrum Access context and we are able to prove not only the existence of a Generalized Nash Equilibrium (GNE), but also its uniqueness; we also show the convergence of a Best Response-type algorithm to the unique GNE. The Nash Equilibrium is said Generalized because we consider a Game in which the strategy sets of the players depend on the choices of the other players [29].

The conditions we propose are easy to be satisfied by users, as we will see in the following, because users do not need to know every single power allocated by all the other users, information generally not available, but they just need to have a locally available information, i.e. the total interference they experience near their own 
receiver.

The paper is organized as follows: in Section 2 the model is described; in Section 3 the mathematical Game is given; in Section 4 conditions ensuring existence and uniqueness of the Generalized Nash Equilibrium are given and a theoretic analysis is discussed. In Section 5 we remark some conclusions.

\section{The Model}

The model concerns a frequency band where one or more primary users, owners of the band's rights, and secondary users coexist in a Dynamic Spectrum Access (DSA) context. Primary users should not suffer a degradation of their own signal because of the presence of the secondary users. More precisely, primary users should not be aware of the presence of the secondary ones in their own frequency band. This is the reason for which such a model is not a cooperative one. Primary users select an interference considered reasonable on the shared channel and fix the value of total tolerable interference. Such a value represents an upper bound for the total transmit power for the secondary users, therefore secondary users have a maximum transmission power imposed by the primary ones. Each secondary user selects its transmission power in order to achieve the best signal quality, ensuring low interference to the primary users and to the other secondary ones.

Suppose there are $N \geq 2$ secondary users and $L \geq 1$ primary ones. Say $\mathscr{N}=$ $\{1, \ldots, N\}$ the set of indexes for the secondary users and $\mathscr{L}=\{1, \ldots, L\}$ the set of indexes for the primary ones. Each secondary user is characterized by a transmitter site $T X_{i}^{s}$ and a receiver site $R X_{i}^{s}$ with $i \in \mathscr{N}$. Similarly, each primary user is characterized by a transmitter site $T X_{i}^{p}$ and a receiver site $R X_{i}^{p}$ with $i \in \mathscr{L}$. The positions of transmitters and receivers are independent of each others.

We recall that a signal transmitted by the $i$-th (primary or secondary) transmitter to the $i$-th receiver produces some interference at the $j$-th receiver, for every $j \neq i$ primary or secondary. Say $g_{i, j}$ the channel gain between the $i$-th secondary transmitter and the $j$-th secondary receiver. If $p_{i}$ is the transmission power (in Watt) of the $i$-th secondary user, its receiver gets a signal $g_{i, i} p_{i}$, while all the other secondary users $j \neq i$ experience an interference $g_{i, j} p_{i}$ due to the $i$-th user. Similarly, say $g_{m, j}^{1,2}$ the channel gain between the $m$-th primary user and the $j$-th secondary one. Say $P_{m}^{\prime}>0$ the (constant) power transmitted by the primary user $m$.

Let us consider the total interference plus noise $I_{i}^{r}$ received by the $i$-th secondary user: it is due to both secondary and primary transmissions and to $\sigma_{i}^{2}$ (in Watt), the 
Additive White Gaussian Noise (AWGN) at the $i$-th receiver $R X_{i}^{s}$. It is:

$$
I_{i}^{r}:=\sum_{k=1, k \neq i}^{N} g_{k, i} p_{k}+\sigma_{i}^{2}+\sum_{m=1}^{L} g_{m, i}^{1,2} P_{m}^{\prime} .
$$

Let us recall the definition of the Signal to Interference plus Noise Ratio (SINR) $\gamma_{i}$ for the $i$-th secondary user: it is the ratio between the signal (transmitted from the $i$-th transmitter $T X_{i}^{s}$ ) received by the $i$-th receiver $R X_{i}^{s}$ and the total interference plus noise that $R X_{i}^{s}$ receives:

$$
\gamma_{i}:=\frac{g_{i, i} p_{i}}{\sum_{k=1, k \neq i}^{N} g_{k, i} p_{k}+\sigma_{i}^{2}+\sum_{m=1}^{L} g_{m, i}^{1,2} P_{m}^{\prime}} .
$$

By putting Eq. (1) in Eq. (2), $\gamma_{i}$ becomes:

$$
\gamma_{i}=\frac{g_{i, i} p_{i}}{I_{i}^{r}}
$$

Say $f\left(\gamma_{i}\right)$ the efficiency function: it represents the probability of correct reception of a signal at a receiver. The efficiency function is defined as:

$$
f\left(\gamma_{i}\right)=\left(1-2 P_{e}\left(\gamma_{i}\right)\right)^{M}
$$

where $P_{e}$ is the bit error rate (BER), see [25], and $M$ is the total number of bits per packet. In general, the efficiency function is strictly increasing and it is such that $\lim _{\gamma_{i} \rightarrow 0} f\left(\gamma_{i}\right)=0$ and $\lim _{\gamma_{i} \rightarrow+\infty} f\left(\gamma_{i}\right)=1$. In [25] the following expressions are given for $P_{e}$, depending on the different modulation schemes: $P_{e}\left(\gamma_{i}\right)=Q\left(\sqrt{2 \gamma_{i}}\right)$ for Binary Phase-Shift Keying (BPSK), $P_{e}\left(\gamma_{i}\right)=\frac{1}{2} \mathrm{e}^{-\gamma_{i}}$ for Differential Phase-Shift Keying (DPSK), $P_{e}\left(\gamma_{i}\right)=Q\left(\sqrt{\gamma_{i}}\right)$ for Coherent Frequency-Shift Keying (Coherent FSK) and $P_{e}\left(\gamma_{i}\right)=\frac{1}{2} \mathrm{e}^{-\gamma_{i} / 2}$ for Non-coherent Frequency-Shift Keying (Non-coherent FSK). Function $Q$ is related to the complementary error function erfc as follows: $Q(x)=$ $\frac{1}{2} \operatorname{erfc}\left(\frac{x}{\sqrt{2}}\right)$. In [30] it is also assumed $P_{e}\left(\gamma_{i}\right)=\frac{1}{2} \mathrm{e}^{-c \gamma_{i}}$ for $c>0$, that is a generic expression that represents the BER of many modulation schemes, for instance when $c=\frac{1}{2}$ or $c=1$ one obtains the Non-coherent FSK and the DPSK schemes respectively.

Our model is suitable for all of these cases, with $M>2$ in (4). For $M \in[1,2]$ the model is not very significant, because the Nash Equilibrium is reached by users always transmitting at an admissible minimum power. It is often assumed that $M=80$.

We assume $f\left(\gamma_{i}\right)$ strictly increasing, with $\lim _{\gamma_{i} \rightarrow 0} f\left(\gamma_{i}\right)=0$ and $\lim _{\gamma_{i} \rightarrow+\infty} f\left(\gamma_{i}\right)=$ 1 , and $f^{\prime \prime}\left(\gamma_{i}\right)>0$ for every $\gamma_{i}<\gamma_{M}, f^{\prime \prime}\left(\gamma_{i}\right)=0$ for $\gamma_{i}=\gamma_{M}$ and $f^{\prime \prime}\left(\gamma_{i}\right)<0$ for every $\gamma_{i}>\gamma_{M}$ for a suitable $\gamma_{M}>0$. For BPSK and Coherent FSK modulation schemes, the value of $\gamma_{M}$ can be computed numerically. For $P_{e}\left(\gamma_{i}\right)=\frac{1}{2} \mathrm{e}^{-c \gamma_{i}}$ with $c>0$, it is 
$\gamma_{M}=\frac{1}{c} \ln M$. With these assumptions, the graph of function $g\left(\gamma_{i}\right):=\gamma_{i} f^{\prime}\left(\gamma_{i}\right)-f\left(\gamma_{i}\right)$, whose derivative with respect to $\gamma_{i}$ is $\gamma_{i} f^{\prime \prime}\left(\gamma_{i}\right)$, for positive values of $\gamma_{i}$ acts as follows: it starts from the origin of the cartesian axes, strictly increases for $\gamma_{i} \in\left(0, \gamma_{M}\right)$, reaches its local and global maximum for $\gamma_{i}=\gamma_{M}$ and monotonically decreases for $\gamma_{i}>\gamma_{M}$, tending to -1 for $\gamma_{i} \rightarrow+\infty$.

\section{The Generalized Nash Equilibrium Problem: Players and Utilities}

Let us introduce our Generalized Nash Equilibrium Problem (GNEP), a non cooperative game where the strategy set of each player depends on the strategies of the other players; the concept of GNEP has been formally introduced in [29] with the name of "Abstract Economy", see also [31]; a complete overview can be found in [32]. In the following subsection, we recall some preliminary concepts.

\subsection{Preliminaries}

In what follows, we adopt the standard notation in Game Theory for which a vector $\boldsymbol{a}=\left(a_{1}, \ldots, a_{N}\right)$ is represented by expression $\boldsymbol{a}=\left(a_{i}, \boldsymbol{a}_{-i}\right)$ in order to underline the choice $a_{i}$ of the $i$-th player with respect to the choices of the other $N-1$ players, represented by index $-i$.

A GNEP is represented by a set $\mathscr{N}$ of $N \geq 2$ players, each player controlling the variable $a_{i}$, that belongs to strategy set $A_{i}$ for every $i \in \mathscr{N}$. The strategy sets $A_{i}$ depend on the other players' strategies: $A_{i}=A_{i}\left(\boldsymbol{a}_{-i}\right)$ for every $i \in \mathscr{N}$. This is the peculiarity of GNEPs: if the strategy sets are independent from the strategies of the other players, then GNEP reduces to a Nash Equilibrium Problem (NEP) or non-cooperative Game, see [33], [34] and [35] to examine Game Theory in depth.

In GNEPs, like in NEPs, every player aims at maximizing an objective function, or utility function, $u_{i}$, which depends on players' action vectors.

Definition 1. A GNEP consists in finding a feasible action vector $\boldsymbol{a}^{*}=\left(a_{1}^{*}, \ldots, a_{N}^{*}\right)=$ $\left(a_{i}^{*}, \boldsymbol{a}_{-i}^{*}\right)$ with $a_{i}^{*} \in A_{i}\left(\boldsymbol{a}_{-i}^{*}\right)$ for every $i \in \mathscr{N}$ solving problem

$$
\underset{a_{i}}{\operatorname{maximize}} u_{i}\left(a_{i}, \boldsymbol{a}_{-i}\right) \quad \text { subject to } a_{i} \in A_{i}\left(\boldsymbol{a}_{-i}\right) \quad \text { for all } i \in \mathscr{N} \text {. }
$$

Definition 2. A Generalized Nash Equilibrium (GNE) is a vector $\boldsymbol{a}^{*}=\left(a_{i}^{*}, \boldsymbol{a}_{-i}^{*}\right) \in$ $A\left(\boldsymbol{a}^{*}\right)$ such that

$$
a_{i}^{*} \in \arg \max _{a_{i} \in A_{i}\left(\boldsymbol{a}_{-i}^{*}\right)} u_{i}\left(a_{i}, \boldsymbol{a}_{-i}^{*}\right) \quad \text { for every } i \in \mathscr{N} .
$$


It means that, at a GNE, the strategy of each player is the Best Response to the strategies played by the other ones. Let us recall the concept of Best Response, defined as follows $(\mathscr{P}(X)$ is the power set of set $X)$ :

Definition 3. The Best Response $B R_{i}$ of player $i$ to the strategy $\boldsymbol{a}_{-i} \in A_{-i}$ of the other $N-1$ players is the correspondence $B R_{i}: A_{-i} \rightarrow \mathscr{P}\left(A_{i}\right)$ such that

$$
B R_{i}\left(\boldsymbol{a}_{-i}\right):=\arg \max _{a_{i} \in A_{i}} u_{i}\left(a_{i}, \boldsymbol{a}_{-i}\right)
$$

we also define:

$$
B R(\boldsymbol{a}):=\left(B R_{i}\left(\boldsymbol{a}_{-i}\right)\right)_{i \in \mathscr{N}} .
$$

The Best Response set is not necessarily a singleton. An equivalent formulation for a GNE is therefore:

Definition 4. A GNE is a vector $\boldsymbol{a}^{*}=\left(a_{i}^{*}, \mathbf{a}_{-i}^{*}\right) \in A\left(\boldsymbol{a}^{*}\right)$ such that $a_{i}^{*} \in B R_{i}\left(\boldsymbol{a}_{-i}^{*}\right)$ for every $i \in \mathscr{N}$; we can write $\boldsymbol{a}^{*} \in B R\left(\boldsymbol{a}^{*}\right)$, that is $\boldsymbol{a}^{*}$ is a fixed point for $B R(\boldsymbol{a})$.

Powerful updating algorithms are based on Best Responses, see [36] and [37].

\subsection{The GNEP}

In this Section we propose a GNEP for power control for secondary users in a frequency band where the primary ones are transmitting. Players are $N$ secondary users. In this model, note that the $L$ primary users in the frequency band are not players: they are rather a constraint.

Following [26], [27] and [28], we assume that in the game there is an exogenous parameter $\omega=(\beta, \delta, \mu) \in \mathbb{R}^{3}$. This parameter is called exogenous because players do not control it. We can tune it in order to adapt to the environment and to obtain the desired outcomes. As it will be clearer in the following, parameter $\beta$ represents the maximum pricing value, $\delta$ is the price weight of the generated interference; $\mu$ is the sensitivity of users to interference, see [26]. In [26] and [27] it is assumed that $\beta \geq \delta>0$ and $\mu>1$. But we assume that the triple $(\beta, \delta, \mu)$ belongs to the set $\Omega:=\left\{(\beta, \delta, \mu) \in \mathbb{R}^{3} \mid \beta \geq \delta>0,0<\mu<\mu^{*}\right\} \subset \mathbb{R}^{3}$ where $\mu^{*}$ is defined in Eq. (8). Our assumptions

$$
\begin{gathered}
\beta \geq \delta>0 \\
0<\mu<\mu^{*}:=\min _{i \in \mathscr{N}}\left\{2 g_{i i}\left(\gamma_{M} \sum_{k=1, k \neq i}^{N} g_{i, k}\right)^{-1}\right\}
\end{gathered}
$$

will be clearer in the following mathematical treatment. 
For every $i \in \mathscr{N}$, the strategy set $P_{i}$ is constituted by the transmitting powers $p_{i}$ that player $i$ can choose; it is assumed to be

$$
P_{i}\left(\mathbf{p}_{-i}\right):=\left[p_{i, \min }\left(\mathbf{p}_{-i}\right), p_{i, \max }\left(\mathbf{p}_{-i}\right)\right] \subset \mathbb{R}^{+},
$$

where

$$
\begin{aligned}
& p_{i, \min }\left(\mathbf{p}_{-i}\right):=\min \left\{\frac{\gamma_{M}}{g_{i, i}} I_{i}^{r}\left(\mathbf{p}_{-i}\right), P_{i, \max }\right\}>0, \\
& p_{i, \max }\left(\mathbf{p}_{-i}\right):=\min \left\{\frac{2 I_{i}^{r}\left(\mathbf{p}_{-i}\right)}{\mu \sum_{k=1, k \neq i}^{N} g_{i, k}}, P_{i, \max }\right\},
\end{aligned}
$$

$P_{i, \max }$ being the maximum power that transmitter $T X_{i}^{s}$ can transmit at. Because of Eq. (8) it is $\gamma_{M}\left(g_{i, i}\right)^{-1} I_{i}^{r}\left(\mathbf{p}_{-i}\right) \leq 2 I_{i}^{r}\left(\mathbf{p}_{-i}\right)\left(\mu \sum_{k=1, k \neq i}^{N} g_{i, k}\right)^{-1}$ and therefore $p_{i, \min }\left(\mathbf{p}_{-i}\right)$ $\leq p_{i, \max }\left(\mathbf{p}_{-i}\right)$ for every $i \in \mathscr{N}$. According to Eq. (11), the more the set of users is sensitive to interference (sensitivity is represented by $\mu$ ), the smaller the maximum power users can select is. As a consequence of the choice of the strategy sets (9), it is:

$$
\gamma_{M} \leq \gamma_{i} \leq \gamma_{\mu}^{i}:=\frac{2 g_{i, i}}{\mu \sum_{k=1, k \neq i}^{N} g_{i, k}}
$$

Following [37], we give the next two definitions for the constrained strategy sets:

Definition 5. Let $A$ and $B$ be sublattices of a lattice $X$. We say that $A$ precedes $B$, $A \prec B$, if for every $a \in A$ and $b \in B, \min \{a, b\} \in A$ and $\max \{a, b\} \in B$.

Definition 6. Consider the constrained strategy set $A_{i}$ for $i \in \mathscr{N}$. If $\mathbf{a}_{-i} \leq \mathbf{a}_{-i}^{\prime}$ implies $A_{i}\left(\mathbf{a}_{-i}\right) \prec A_{i}\left(\mathbf{a}_{-i}^{\prime}\right)$, then the set $A_{i}$ has the ascending property.

Therefore we can state that:

Remark 1. The strategy set $P_{i}\left(\mathbf{p}_{-i}\right)$ in (9) has the ascending property for every $i \in \mathscr{N}$.

The set $P=P_{1} \times \ldots \times P_{N}=P_{i} \times P_{-i}$ is the strategy space relative to the $N$ players; vectors of powers $\mathbf{p}=\left(p_{1}, \ldots, p_{N}\right)=\left(p_{i}, \mathbf{p}_{-i}\right) \in P$ are the strategies.

In the game, each player will choose its own transmission power in order to maximize its own utility function. In a previous work, see [28], we have modeled a game for Centralized Dynamic Spectrum Leasing (C-DSL) for secondary and primary users, following [27] for the secondary utility, but with the introduction of multiple primary 
users and overall assuming that the secondary utility of player $i$ depends on simultaneous choices of players (like in [25]) instead of on their previous choices. In this game, we use the same expression for the utility of secondary users assumed in [28]. In the present paper, we focus only on secondary players, assuming that the primary ones are not playing the game, in a DSA context. Anyway, results regarding the secondary utilities could be easily employed also in the C-DSL context of [28], but this is not the tool of the present paper.

Hence, the expression in bit/Joule for the utilities $\left\{u_{i}^{\omega}\right\}_{i \in \mathscr{N}}$, where $u_{i}^{\omega}: P \times \Omega \rightarrow \mathbb{R}$, is defined as follows:

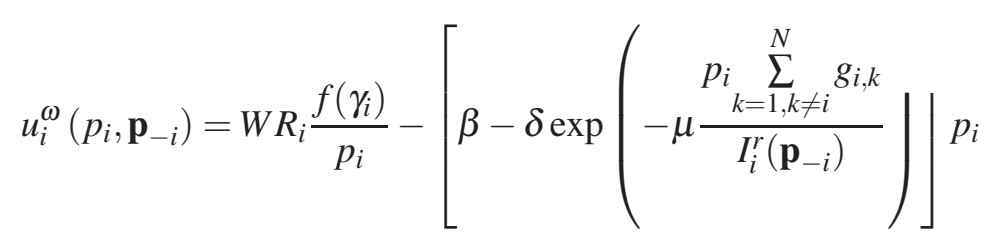

where $W$ is the ratio between the number of information bits per packet and the total number of bits per packet transmitted by the users and $R_{i}$ is the transmission rate of the $i$-th user (in bits/sec).

Say

$$
\Delta\left(p_{i}, I_{i}^{r}\left(\mathbf{p}_{-i}\right)\right):=\left[\beta-\delta \exp \left(\begin{array}{c}
p_{i} \sum_{k=1, k \neq i}^{N} g_{i, k} \\
-\mu \frac{I_{i}^{r}\left(\mathbf{p}_{-i}\right)}{}
\end{array}\right)\right] p_{i}
$$

the so called pricing function. The quantity in the square brackets of Eq. (13) (or equivalently Eq. (14)) is called pricing factor and it is assumed to have units $\frac{\text { bit }}{(\text { Watt })^{2} \times \mathrm{sec}}$ so that the utility function has a consistent unit of measurement.

The pricing function is such that the higher the interference generated by a user transmitting at a high power is, the greater the value of pricing it will have to pay is. Indeed, consider $p_{i}>p_{i}^{\prime}$ both in $P_{i}$. For $I_{i}^{r}\left(\mathbf{p}_{-i}\right)$ fixed, it results:

$$
\beta-\delta \exp \left(-\mu \frac{p_{i} \sum_{k=1, k \neq i}^{N} g_{i, k}}{I_{i}^{r}\left(\mathbf{p}_{-i}\right)}\right)>\beta-\delta \exp \left(\begin{array}{c}
p_{i}^{\prime} \sum_{k=1, k \neq i}^{N} g_{i, k} \\
\left.-\mu \frac{I_{i}^{r}\left(\mathbf{p}_{-i}\right)}{}\right)
\end{array}\right)
$$


leading to

$$
\Delta\left(p_{i}, I_{i}^{r}\left(\mathbf{p}_{-i}\right)\right)>\left[\beta-\delta \exp \left(-\mu \frac{p_{i}^{\prime} \sum_{k=1, k \neq i}^{N} g_{i, k}}{I_{i}^{r}\left(\mathbf{p}_{-i}\right)}\right)\right] p_{i}>\Delta\left(p_{i}^{\prime}, I_{i}^{r}\left(\mathbf{p}_{-i}\right)\right) .
$$

Therefore, the presence of a pricing function in the utility function implicitly induces a sort of cooperation, yet maintaining the non-cooperative nature of such a game.

Besides, the higher the interference $I_{i}^{r}\left(\mathbf{p}_{-i}\right)$ received by the $i$-th user is, the smaller the pricing factor for the $i$-th user is. It implicitly involves that if user $i$ receives a great interference, user $i$ is in some way allowed to use a power higher than the one it would otherwise use, in the sense that it can raise its power without paying an excessive price.

Note that for $p_{i} \in P_{i}$ as defined in (9) the pricing factor is not greater than $\beta-\frac{\delta}{\mathrm{e}^{2}}$ (instead of $\beta$, like it was in [26]), while it is still greater than $\beta-\delta$. The presence of the sensitivity $\mu$ in the upper bound of $p_{i, \max }\left(\mathbf{p}_{-i}\right)$ lets the game impose a lower maximum pricing to players.

Remark 2. In [28] we assume a smaller interval for the set of powers, indeed it is assumed that the minimum allowed power is the same, but the maximum allowed power is

$$
\tilde{p}_{i, \max }\left(\mathbf{p}_{-i}\right):=\min \left\{\frac{I_{i}^{r}\left(\mathbf{p}_{-i}\right)}{\mu \sum_{k=1, k \neq i}^{N} g_{i, k}}, P_{i, \max }\right\} .
$$

This smaller interval ensures the Supermodularity of the GNEP. Supermodular games have remarkable properties, see [37], [38], [39], [40], [41], [42] and [43]. In the present paper we enlarge the strategy sets in order to obtain more general results, valid even outside the supermodularity interval.

\section{Generalized Nash Equilibrium}

In the present Section we show the existence and the uniqueness of the Generalized Nash Equilibrium (GNE) for GNEP proposed in Section 3. 


\subsection{Existence of a GNE}

In order to prove the existence of a Generalized Nash Equilibrium, we follow a major result by [31]. A simplified version of such a theorem, given in [44], is here reported for a maximization GNEP.

Theorem 7 ([44]). Consider a GNEP with $N$ players in which:

1. there exist $N$ non-empty, convex and compact sets $K_{i} \subseteq \mathbb{R}^{n_{i}}$ such that for every $\boldsymbol{p}=\left(p_{i}, \boldsymbol{p}_{-i}\right) \in \mathbb{R}^{n}$, where $n:=\sum_{i=1} n_{i}$, with $p_{i} \in K_{i}$ for every $i \in \mathscr{N}$, the strategy sets $P_{i}\left(\boldsymbol{p}_{-i}\right)$ are non-empty, closed and convex and such that $P_{i}\left(\boldsymbol{p}_{-i}\right) \subseteq K_{i}$;

2. for every $i \in \mathscr{N}, P_{i}(\cdot)$ is a lower semicontinuous and an upper semicontinuous point-to-set map;

3. for every $i \in \mathscr{N}$, the utility function $u_{i}$ is continuous;

4. for every $i \in \mathscr{N}$, the utility function $u_{i}\left(\cdot, \boldsymbol{p}_{-i}\right)$ is quasi-concave in $p_{i}$ on $P_{i}\left(\boldsymbol{p}_{-i}\right)$; therefore a GNE exists.

In the following we show that assumptions in the previous theorem hold for our GNEP. The proof is almost the same given in [28], therefore we just give some hints.

Theorem 8 (Existence of a GNE). The Generalized Nash Equilibrium Problem defined in Section 3 has a Generalized Nash Equilibrium.

Proof. Say $K_{i} \subset \mathbb{R}$ the following compact, convex, non-empty interval for $i \in \mathscr{N}$ : $K_{i}:=\left[0, P_{i, \text { max }}\right]$. It is trivially $P_{i}\left(\mathbf{p}_{-i}\right)$ non-empty, closed and convex and $P_{i}\left(\mathbf{p}_{-i}\right) \subseteq K_{i}$ for every $i \in \mathscr{N}$. Furthermore, for every $i \in \mathscr{N}, P_{i}(\cdot)$ is a lower and upper semicontinuous point-to-set map, because of the continuity of functions $p_{i, \min }\left(\mathbf{p}_{-i}\right)$ and $p_{i, \max }\left(\mathbf{p}_{-i}\right)$. The utility functions $u_{i}^{\omega}$ are trivially continuous for every $i \in \mathscr{N}$. In order to complete the proof of existence af a GNE, one has to show that for every $i \in \mathscr{N}$, the utility function $u_{i}^{\omega}\left(\cdot, \mathbf{p}_{-i}\right)$ is quasi-concave in $p_{i}$ on $P_{i}\left(\mathbf{p}_{-i}\right)$. Let us compute the first derivative of the utility function $u_{i}^{\omega}$ with respect to $p_{i}$ for every $i \in \mathscr{N}$, for $\mathbf{p}_{-i}$ fixed:

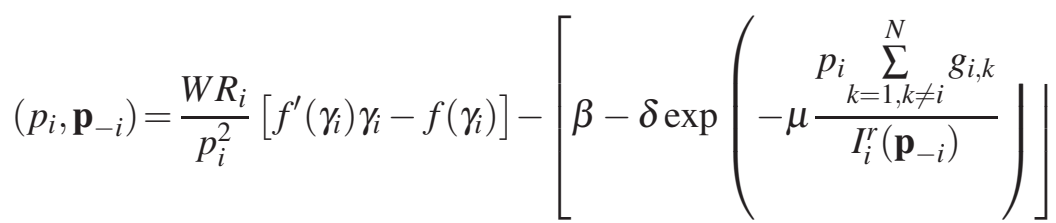

$$
\begin{aligned}
& -\left[\delta \mu \frac{\sum_{k=1, k \neq i}^{N} g_{i, k}}{I_{i}^{r}\left(\mathbf{p}_{-i}\right)} \exp \left(-\mu \frac{p_{i} \sum_{k=1, k \neq i}^{N} g_{i, k}}{I_{i}^{r}\left(\mathbf{p}_{-i}\right)}\right)\right] p_{i} .
\end{aligned}
$$


By equaling the right hand side of Eq. (16) to zero, we put a necessary condition for $p_{i}$ to be a local maximum point for $u_{i}^{\omega}\left(p_{i}, \mathbf{p}_{-i}\right)$ for a fixed vector $\mathbf{p}_{-i}$. It is

$$
\begin{aligned}
& f^{\prime} \quad\left(\frac{g_{i, i} p_{i}}{I_{i}^{r}\left(\mathbf{p}_{-i}\right)}\right) \frac{g_{i, i} p_{i}}{I_{i}^{r}\left(\mathbf{p}_{-i}\right)}-f\left(\frac{g_{i, i} p_{i}}{I_{i}^{r}\left(\mathbf{p}_{-i}\right)}\right) \\
& =\frac{1}{W R_{i}}\left[\beta-\delta \exp \left(\begin{array}{c}
p_{i} \sum_{k=1, k \neq i}^{N} g_{i, k} \\
I_{i}^{r}\left(\mathbf{p}_{-i}\right)
\end{array}\right) \mid p_{i}^{2}\right. \\
& +\frac{1}{W R_{i}}\left[\delta \mu \frac{\sum_{k=1, k \neq i}^{N} g_{i, k}}{I_{i}^{r}\left(\mathbf{p}_{-i}\right)} \exp \left(\begin{array}{c}
p_{i} \sum_{k=1, k \neq i}^{N} g_{i, k} \\
I_{i}^{r}\left(\mathbf{p}_{-i}\right)
\end{array}\right)\right] p_{i}^{3} .
\end{aligned}
$$

We solve the transcendental equation (17) by a graphical method. Say

$$
\begin{aligned}
& \operatorname{LHS}\left(p_{i}, I_{i}^{r}\left(\mathbf{p}_{-i}\right)\right):=f^{\prime}\left(\frac{g_{i, i} p_{i}}{I_{i}^{r}\left(\mathbf{p}_{-i}\right)}\right) \frac{g_{i, i} p_{i}}{I_{i}^{r}\left(\mathbf{p}_{-i}\right)}-f\left(\frac{g_{i, i} p_{i}}{I_{i}^{r}\left(\mathbf{p}_{-i}\right)}\right), \\
& \operatorname{RHS}\left(p_{i}, I_{i}^{r}\left(\mathbf{p}_{-i}\right)\right):=\frac{1}{W R_{i}}\left[\beta-\delta \exp \left(-\mu \frac{p_{i} \sum_{k=1, k \neq i}^{N} g_{i, k}}{I_{i}^{r}\left(\mathbf{p}_{-i}\right)}\right) \mid p_{i}^{2}\right. \\
& +\frac{1}{W R_{i}}\left[\delta \mu \frac{\sum_{k=1, k \neq i}^{N} g_{i, k}}{I_{i}^{r}\left(\mathbf{p}_{-i}\right)} \exp \left(-\mu \frac{p_{i} \sum_{k=1, k \neq i}^{N} g_{i, k}}{I_{i}^{r}\left(\mathbf{p}_{-i}\right)}\right) \mid p_{i}^{3} .\right.
\end{aligned}
$$

Function $\operatorname{LHS}\left(p_{i}, I_{i}^{r}\left(\mathbf{p}_{-i}\right)\right)$ is monotonically decreasing in the domain $P_{i}\left(\mathbf{p}_{-i}\right)$, with $\operatorname{LHS}\left(p_{i, \min }\left(\mathbf{p}_{-i}\right), I_{i}^{r}\left(\mathbf{p}_{-i}\right)\right)>0$.

Function $\operatorname{RHS}\left(p_{i}, I_{i}^{r}\left(\mathbf{p}_{-i}\right)\right)$ is strictly positive and monotonically increasing in $P_{i}\left(\mathbf{p}_{-i}\right)$, see conditions (7) and (8).

Three cases are possible: $\operatorname{LHS}\left(p_{i}, I_{i}^{r}\left(\mathbf{p}_{-i}\right)\right)>\operatorname{RHS}\left(p_{i}, I_{i}^{r}\left(\mathbf{p}_{-i}\right)\right)$ for every $p_{i} \in$ $P_{i}\left(\mathbf{p}_{-i}\right)$ implying that function $u_{i}^{\omega}$ is strictly increasing (and the unique global maximum point is $\left.p_{i}=p_{i, \max }\left(\mathbf{p}_{-i}\right)\right)$, or $\operatorname{LHS}\left(p_{i}, I_{i}^{r}\left(\mathbf{p}_{-i}\right)\right)<R H S\left(p_{i}, I_{i}^{r}\left(\mathbf{p}_{-i}\right)\right)$ for every $p_{i} \in P_{i}\left(\mathbf{p}_{-i}\right)$, that is $u_{i}^{\omega}$ strictly decreasing (and the unique global maximum point is $p_{i}=p_{i, \min }\left(\mathbf{p}_{-i}\right)$ ), or finally $\operatorname{LHS}\left(p_{i}, I_{i}^{r}\left(\mathbf{p}_{-i}\right)\right)=R H S\left(p_{i}, I_{i}^{r}\left(\mathbf{p}_{-i}\right)\right)$ for one (and only one) $p_{i} \in P_{i}\left(\mathbf{p}_{-i}\right)$. Such a power value is a local maximum point and it is the unique 
global maximum point. Therefore for every $i \in \mathscr{N}$ the secondary utility $u_{i}\left(\cdot, \mathbf{p}_{-i}\right)$ is quasi-concave in $p_{i}$ on $P_{i}\left(\mathbf{p}_{-i}\right)$.

All the assumptions of Theorem 7 are therefore fulfilled and a GNE exists.

\subsection{Uniqueness of the GNE}

In [36], in a power control context, the property of scalability for a function defined on positive vectors has been introduced, in order to define a standard function as follows:

Definition 9. A function $\mathbf{X}(\cdot)$ defined on positive vectors $\mathbf{x}$ is a standard function if for every $\mathbf{x}>0$ it has the following properties:

(i) positivity: $\mathbf{X}(\mathbf{x})>0$;

(ii) monotonicity: if $\mathbf{x}^{\prime} \geq \mathbf{x}$, then $\mathbf{X}\left(\mathbf{x}^{\prime}\right) \geq \mathbf{X}(\mathbf{x})$;

(iii) scalability: for every $\alpha>1, \alpha \mathbf{X}(\mathbf{x})>\mathbf{X}(\alpha \mathbf{x})$.

Actually, it has been proved [45] that positivity is implied by monotonicity and scalability, $x$ being positive.

In order to prove the uniqueness of the Generalized Nash Equilibrium, we use the concept of Best Response recalled in Definition 3. Indeed, if the Best Response $B R(\mathbf{p})=\left(B R_{i}\left(\mathbf{p}_{-i}\right)\right)_{i \in \mathscr{N}}$ of a non-cooperative game is a standard function requiring $B R_{i}\left(\mathbf{p}_{-i}\right)$ be single-valued, and provided that a Nash Equilibrium exists for the game, then the Nash Equilibrium is unique and the Best Response Algorithm converges to it, [36] and [37]. The same approach for showing the uniqueness of a GNEP is followed for instance in [46] and [47].

The following steps are therefore intended to prove that the Best Response has properties of monotonicity and scalability. Positivity is trivial, see [45].

Let us prove the following preliminary Lemma.

Lemma 10. The total interference plus noise received by the $i$-th user $I_{i}^{r}\left(\boldsymbol{p}_{-i}\right)$ defined in Eq. (1) is a Standard Function.

Proof. Following Definition 9, we have to prove positivity, monotonicity and scalability of the Interference function $I_{i}^{r}\left(\mathbf{p}_{-i}\right)$ defined in (1) for positive power vectors:

(i) positivity: for every $\mathbf{p}>0$, trivially $I_{i}^{r}\left(\mathbf{p}_{-i}\right)>0$;

(ii) monotonicity: if $\mathbf{p}^{\prime} \geq \mathbf{p}>0$, that is $p_{j}^{\prime} \geq p_{j}>0$ for every $j \in \mathscr{N}$, trivially $I_{i}^{r}\left(\mathbf{p}_{-i}^{\prime}\right) \geq I_{i}^{r}\left(\mathbf{p}_{-i}\right)$ 
(iii) scalability: for every $\alpha>1, \mathbf{p}>0$,

$$
\begin{aligned}
I_{i}^{r}\left(\alpha \mathbf{p}_{-i}\right) & =\sum_{k=1, k \neq i}^{N} g_{k, i} \alpha p_{k}+\sigma_{i}^{2}+\sum_{m=1}^{L} g_{m, i}^{1,2} P_{m}^{\prime} \\
& <\sum_{k=1, k \neq i}^{N} g_{k, i} \alpha p_{k}+\alpha \sigma_{i}^{2}+\alpha \sum_{m=1}^{L} g_{m, i}^{1,2} P_{m}^{\prime}=\alpha I_{i}^{r}\left(\mathbf{p}_{-i}\right)
\end{aligned}
$$

that is

$$
I_{i}^{r}\left(\alpha \mathbf{p}_{-i}\right)<\alpha I_{i}^{r}\left(\mathbf{p}_{-i}\right)
$$

for every $\alpha>1, \mathbf{p}>0$.

The Interference function is therefore a standard function.

As already stated, every player aims at maximizing its utility function $u_{i}^{\omega}\left(p_{i}, \mathbf{p}_{-i}\right)$, basing on the choices of the other players. A necessary condition for a local maximum point for $u_{i}^{\omega}\left(p_{i}, \mathbf{p}_{-i}\right)$ for a fixed vector $\mathbf{p}_{-i}$ is given in Eq. (17).

Because it is not possible to find an explicit solution for Eq. (17), it is hard to show the monotonicity and the scalability of the Best Response. Anyway, as already shown, we can solve the transcendental equation (17) by a graphical method.

In the previous Section we have shown that one can find a unique power $p_{i} \in$ $P_{i}\left(\mathbf{p}_{-i}\right)$ which maximizes $u_{i}^{\omega}$ for a given $\mathbf{p}_{-i}$. Therefore, the Best Response for player $i$ defined in Definition 3 is a singleton and

$$
B R_{i}\left(\mathbf{p}_{-i}\right)=\arg \max _{p_{i} \in P_{i}\left(\mathbf{p}_{-i}\right)} u_{i}^{\omega}\left(p_{i}, \mathbf{p}_{-i}\right), \forall i \in \mathscr{N} .
$$

The Best Response of user $i$ is therefore $B R_{i}\left(\mathbf{p}_{-i}\right)=p_{i, \min }\left(\mathbf{p}_{-i}\right)$ if $L H S\left(p_{i}, I_{i}^{r}\left(\mathbf{p}_{-i}\right)\right)$ $<\operatorname{RHS}\left(p_{i}, I_{i}^{r}\left(\mathbf{p}_{-i}\right)\right)$ for every $p_{i} \in P_{i}\left(\mathbf{p}_{-i}\right)$, or it is $B R_{i}\left(\mathbf{p}_{-i}\right)=p_{i, \max }\left(\mathbf{p}_{-i}\right)$ if $\operatorname{LHS}\left(p_{i}, I_{i}^{r}\left(\mathbf{p}_{-i}\right)\right)>\operatorname{RHS}\left(p_{i}, I_{i}^{r}\left(\mathbf{p}_{-i}\right)\right)$ for every $p_{i} \in P_{i}\left(\mathbf{p}_{-i}\right)$, otherwise it is the (unique) power $p_{i} \in P_{i}\left(\mathbf{p}_{-i}\right)$ which satisfies Eq. (17) or, more briefly, equation $\operatorname{LHS}\left(p_{i}, I_{i}^{r}\left(\mathbf{p}_{-i}\right)\right)=\operatorname{RHS}\left(p_{i}, I_{i}^{r}\left(\mathbf{p}_{-i}\right)\right)$.

Lemma 11. For every $i \in \mathscr{N}$, the Best Responses $B R_{i}$ in Eq. (21) are standard functions.

Proof. In order to simplify the proof of this Lemma, we temporary assume that the strategy sets $P_{i}$ are not constrained by the maximum allowed power $P_{i, \max }$. Therefore in the first part of this section we assume that

$$
p_{i, \min }\left(\mathbf{p}_{-i}\right):=\frac{\gamma_{M}}{g_{i, i}} I_{i}^{r}\left(\mathbf{p}_{-i}\right),
$$




$$
p_{i, \max }\left(\mathbf{p}_{-i}\right):=\frac{2 I_{i}^{r}\left(\mathbf{p}_{-i}\right)}{\mu \sum_{k=1, k \neq i}^{N} g_{i, k}} .
$$

The constraint for the strategy sets will be re-introduced at the end of this proof. We use the following notation for the Best Responses in the unconstrained case: $B R_{i}^{u}\left(\mathbf{p}_{-i}\right)$. We first prove the monotonicity of the unconstrained Best Responses $B R_{i}^{u}\left(\mathbf{p}_{-i}\right)$ for every $i \in \mathscr{N}$.

Proof. [Monotonicity of the unconstrained Best Responses $B R_{i}^{u}\left(\mathbf{p}_{-i}\right)$.] Consider the power vectors $\mathbf{p}^{\prime} \geq \mathbf{p}$. We are going to prove that $B R_{i}^{u}\left(\mathbf{p}_{-i}^{\prime}\right) \geq B R_{i}^{u}\left(\mathbf{p}_{-i}\right)$ for $i \in \mathscr{N}$. Inequality $\mathbf{p}^{\prime} \geq \mathbf{p}$ implies $\mathbf{p}_{-i}^{\prime} \geq \mathbf{p}_{-i}$ by definition and therefore $I_{i}^{r}\left(\mathbf{p}_{-i}^{\prime}\right) \geq$ $I_{i}^{r}\left(\mathbf{p}_{-i}\right)$ for the monotonicity of the Interference function, see Lemma 10. We wonder what happens to functions $\operatorname{LHS}\left(\theta_{i}, I_{i}^{r}\left(\mathbf{p}_{-i}\right)\right)$ and $\operatorname{RHS}\left(\theta_{i}, I_{i}^{r}\left(\mathbf{p}_{-i}\right)\right)$ when the Interference function increases. Let us consider the behavior of the graph of $\operatorname{LHS}\left(\theta_{i}, I_{i}^{r}\left(\mathbf{p}_{-i}^{\prime}\right)\right)$ with respect to that of $\operatorname{LHS}\left(\theta_{i}, I_{i}^{r}\left(\mathbf{p}_{-i}\right)\right)$. Following Eq. (18), it is:

$$
\begin{aligned}
& \operatorname{LHS}\left(\theta_{i}, I_{i}^{r}\left(\mathbf{p}_{-i}^{\prime}\right)\right):=f^{\prime}\left(\frac{g_{i, i} \theta_{i}}{I_{i}^{r}\left(\mathbf{p}_{-i}^{\prime}\right)}\right) \frac{g_{i, i} \theta_{i}}{I_{i}^{r}\left(\mathbf{p}_{-i}^{\prime}\right)}-f\left(\frac{g_{i, i} \theta_{i}}{I_{i}^{r}\left(\mathbf{p}_{-i}^{\prime}\right)}\right) \\
& =f^{\prime}\left(\frac{g_{i, i} \theta_{i}}{I_{i}^{r}\left(\mathbf{p}_{-i}\right)} \frac{I_{i}^{r}\left(\mathbf{p}_{-i}\right)}{I_{i}^{r}\left(\mathbf{p}_{-i}^{\prime}\right)}\right) \frac{g_{i, i} \theta_{i}}{I_{i}^{r}\left(\mathbf{p}_{-i}\right)} \frac{I_{i}^{r}\left(\mathbf{p}_{-i}\right)}{I_{i}^{r}\left(\mathbf{p}_{-i}^{\prime}\right)}-f\left(\frac{g_{i, i} \theta_{i}}{I_{i}^{r}\left(\mathbf{p}_{-i}\right)} \frac{I_{i}^{r}\left(\mathbf{p}_{-i}\right)}{I_{i}^{r}\left(\mathbf{p}_{-i}^{\prime}\right)}\right) \\
& =\operatorname{LHS}\left(\theta_{i} \frac{I_{i}^{r}\left(\mathbf{p}_{-i}\right)}{I_{i}^{r}\left(\mathbf{p}_{-i}^{\prime}\right)}, I_{i}^{r}\left(\mathbf{p}_{-i}\right)\right) .
\end{aligned}
$$

By putting $c:=\frac{I_{i}^{r}\left(\mathbf{p}_{-i}\right)}{I_{i}^{T}\left(\mathbf{p}_{-i}^{\prime}\right)}$, it is $0<c \leq 1$ and the previous equation becomes:

$$
\operatorname{LHS}\left(\theta_{i}, I_{i}^{r}\left(\mathbf{p}_{-i}^{\prime}\right)\right)=\operatorname{LHS}\left(c \theta_{i}, I_{i}^{r}\left(\mathbf{p}_{-i}\right)\right) .
$$

The case $c=1$ is trivial. If $0<c<1$, the graph of $\operatorname{LHS}\left(\theta_{i}, I_{i}^{r}\left(\mathbf{p}_{-i}^{\prime}\right)\right)$ is a horizontal dilatation of the graph of $\operatorname{LHS}\left(\theta_{i}, I_{i}^{r}\left(\mathbf{p}_{-i}\right)\right)$ by the factor $\frac{1}{c}>1$ (that implies a horizontal stretching of the graph).

Now we wonder what happens to the graph of $\operatorname{RHS}\left(\theta_{i}, I_{i}^{r}\left(\mathbf{p}_{-i}^{\prime}\right)\right)$ with respect to the one of $\operatorname{RHS}\left(\theta_{i}, I_{i}^{r}\left(\mathbf{p}_{-i}\right)\right)$. It can be computed that it is $\operatorname{RHS}\left(\theta_{i}, I_{i}^{r}\left(\mathbf{p}_{-i}^{\prime}\right)\right)<$ $R H S\left(\theta_{i}, I_{i}^{r}\left(\mathbf{p}_{-i}\right)\right)$ if and only if $\theta_{i}<\theta_{0}$ for a value $\theta_{0} \in\left(p_{i, \max }\left(\mathbf{p}_{-i}\right), p_{i, \max }\left(\mathbf{p}_{-i}^{\prime}\right)\right)$.

Consider $B R_{i}^{u}\left(\mathbf{p}_{-i}\right)$. Because of its definition, it belongs to the strategy set $P_{i}\left(\mathbf{p}_{-i}\right)=\left[p_{i, \min }\left(\mathbf{p}_{-i}\right), p_{i, \text { max }}\left(\mathbf{p}_{-i}\right)\right]$. We are going to prove that for $\mathbf{p}_{-i}^{\prime} \geq \mathbf{p}_{-i}$, it is $B R_{i}^{u}\left(\mathbf{p}_{-i}^{\prime}\right) \geq B R_{i}^{u}\left(\mathbf{p}_{-i}\right)$. It is certainly $B R_{i}^{u}\left(\mathbf{p}_{-i}^{\prime}\right) \in P_{i}\left(\mathbf{p}_{-i}^{\prime}\right)=\left[p_{i, \min }\left(\mathbf{p}_{-i}^{\prime}\right), p_{i, \max }\left(\mathbf{p}_{-i}^{\prime}\right)\right]$. Because of the ascending property of the strategy sets, see Remark 1 , it is $p_{i, \min }\left(\mathbf{p}_{-i}\right) \leq$ $p_{i, \min }\left(\mathbf{p}_{-i}^{\prime}\right)$ and $p_{i, \max }\left(\mathbf{p}_{-i}\right) \leq p_{i, \max }\left(\mathbf{p}_{-i}^{\prime}\right)$.

Two cases can occur: 
1. $p_{i, \min }\left(\mathbf{p}_{-i}\right) \leq p_{i, \min }\left(\mathbf{p}_{-i}^{\prime}\right) \leq p_{i, \max }\left(\mathbf{p}_{-i}\right) \leq p_{i, \max }\left(\mathbf{p}_{-i}^{\prime}\right)$;

2. $p_{i, \min }\left(\mathbf{p}_{-i}\right) \leq p_{i, \max }\left(\mathbf{p}_{-i}\right) \leq p_{i, \min }\left(\mathbf{p}_{-i}^{\prime}\right) \leq p_{i, \max }\left(\mathbf{p}_{-i}^{\prime}\right)$.

Let us study the two cases:

2. The second case is the easiest one: indeed it is

$$
\begin{aligned}
p_{i, \min }\left(\mathbf{p}_{-i}\right) & \leq B R_{i}^{u}\left(\mathbf{p}_{-i}\right) \leq p_{i, \max }\left(\mathbf{p}_{-i}\right) \leq p_{i, \min }\left(\mathbf{p}_{-i}^{\prime}\right) \\
& \leq B R_{i}^{u}\left(\mathbf{p}_{-i}^{\prime}\right) \leq p_{i, \max }\left(\mathbf{p}_{-i}^{\prime}\right)
\end{aligned}
$$

and therefore $B R_{i}^{u}\left(\mathbf{p}_{-i}^{\prime}\right) \geq B R_{i}^{u}\left(\mathbf{p}_{-i}\right)$, qed.

1. The first case

$$
p_{i, \min }\left(\mathbf{p}_{-i}\right) \leq p_{i, \min }\left(\mathbf{p}_{-i}^{\prime}\right) \leq p_{i, \max }\left(\mathbf{p}_{-i}\right) \leq p_{i, \max }\left(\mathbf{p}_{-i}^{\prime}\right)
$$

is discussed in the following. There are several possibilities.

(a) Case $\operatorname{LHS}\left(\theta_{i}, I_{i}^{r}\left(\mathbf{p}_{-i}\right)\right)<\operatorname{RHS}\left(\theta_{i}, I_{i}^{r}\left(\mathbf{p}_{-i}\right)\right)$ for every $\theta_{i} \in P_{i}\left(\mathbf{p}_{-i}\right)$, see Fig. 1; therefore it is $B R_{i}^{u}\left(\mathbf{p}_{-i}\right)=p_{i, \min }\left(\mathbf{p}_{-i}\right)$. Because of the ascending property of the strategy sets, it is $p_{i, \min }\left(\mathbf{p}_{-i}\right) \leq p_{i, \min }\left(\mathbf{p}_{-i}^{\prime}\right)$ and therefore it follows $B R_{i}^{u}\left(\mathbf{p}_{-i}\right)=p_{i, \min }\left(\mathbf{p}_{-i}\right) \leq p_{i, \min }\left(\mathbf{p}_{-i}^{\prime}\right) \leq B R_{i}^{u}\left(\mathbf{p}_{-i}^{\prime}\right)$, qed.

(b) Case $\operatorname{LHS}\left(\theta_{i}, I_{i}^{r}\left(\mathbf{p}_{-i}\right)\right)=\operatorname{RHS}\left(\theta_{i}, I_{i}^{r}\left(\mathbf{p}_{-i}\right)\right)$ for one (and only one) $\theta_{i} \in P_{i}\left(\mathbf{p}_{-i}\right)$; therefore it is $\theta_{i}=B R_{i}^{u}\left(\mathbf{p}_{-i}\right)$.

i. If $B R_{i}^{u}\left(\mathbf{p}_{-i}\right) \in\left[p_{i, \min }\left(\mathbf{p}_{-i}\right), p_{i, \min }\left(\mathbf{p}_{-i}^{\prime}\right)\right]$, it is $B R_{i}^{u}\left(\mathbf{p}_{-i}\right) \leq p_{i, \min }\left(\mathbf{p}_{-i}^{\prime}\right) \leq$ $B R_{i}^{u}\left(\mathbf{p}_{-i}^{\prime}\right)$, qed.

ii. If $B R_{i}^{u}\left(\mathbf{p}_{-i}\right) \in\left[p_{i, \min }\left(\mathbf{p}_{-i}^{\prime}\right), p_{i, \max }\left(\mathbf{p}_{-i}\right)\right]$, see Fig.2, we need some considerations. In this interval it is $\operatorname{RHS}\left(\theta_{i}, I_{i}^{r}\left(\mathbf{p}_{-i}^{\prime}\right)\right)<R H S\left(\theta_{i}, I_{i}^{r}\left(\mathbf{p}_{-i}\right)\right)$; say $\theta^{*}$ the abscissa of the point of intersection of the graphs of $\operatorname{LHS}\left(\theta_{i}, I_{i}^{r}\left(\mathbf{p}_{-i}\right)\right)$ and $\operatorname{RHS}\left(\theta_{i}, I_{i}^{r}\left(\mathbf{p}_{-i}^{\prime}\right)\right)$. Therefore it is $\theta^{*}>B R_{i}^{u}\left(\mathbf{p}_{-i}\right)$. Furthermore, $\operatorname{LHS}\left(\theta_{i}, I_{i}^{r}\left(\mathbf{p}_{-i}^{\prime}\right)\right)$ is a horizontal dilation of

$$
\operatorname{LHS}\left(\theta_{i}, I_{i}^{r}\left(\mathbf{p}_{-i}\right)\right)
$$

and the point of intersection of the graphs of $\operatorname{LHS}\left(\theta_{i}, I_{i}^{r}\left(\mathbf{p}_{-i}^{\prime}\right)\right)$ and $R H S\left(\theta_{i}, I_{i}^{r}\left(\mathbf{p}_{-i}^{\prime}\right)\right)$ moves towards right. Therefore it is $B R_{i}^{u}\left(\mathbf{p}_{-i}\right)$ $<\theta^{*}<B R_{i}^{u}\left(\mathbf{p}_{-i}^{\prime}\right)$, qed.

(c) Case $\operatorname{LHS}\left(\theta_{i}, I_{i}^{r}\left(\mathbf{p}_{-i}\right)\right)>\operatorname{RHS}\left(\theta_{i}, I_{i}^{r}\left(\mathbf{p}_{-i}\right)\right)$ for every $\theta_{i} \in P_{i}\left(\mathbf{p}_{-i}\right)$; therefore it is $B R_{i}^{u}\left(\mathbf{p}_{-i}\right)=p_{i, \max }\left(\mathbf{p}_{-i}\right)$. Consider $\theta_{i} \in\left[p_{i, \min }\left(\mathbf{p}_{-i}^{\prime}\right), p_{i, \max }\left(\mathbf{p}_{-i}\right)\right]$. It is $\operatorname{RHS}\left(\theta_{i}, I_{i}^{r}\left(\mathbf{p}_{-i}^{\prime}\right)\right)<\operatorname{RHS}\left(\theta_{i}, I_{i}^{r}\left(\mathbf{p}_{-i}\right)\right)$ because $p_{i, \max }\left(\mathbf{p}_{-i}\right)<\theta_{0}$. Because we are assuming $\operatorname{RHS}\left(\theta_{i}, I_{i}^{r}\left(\mathbf{p}_{-i}\right)\right)<\operatorname{LHS}\left(\theta_{i}, I_{i}^{r}\left(\mathbf{p}_{-i}\right)\right)$ everywhere 


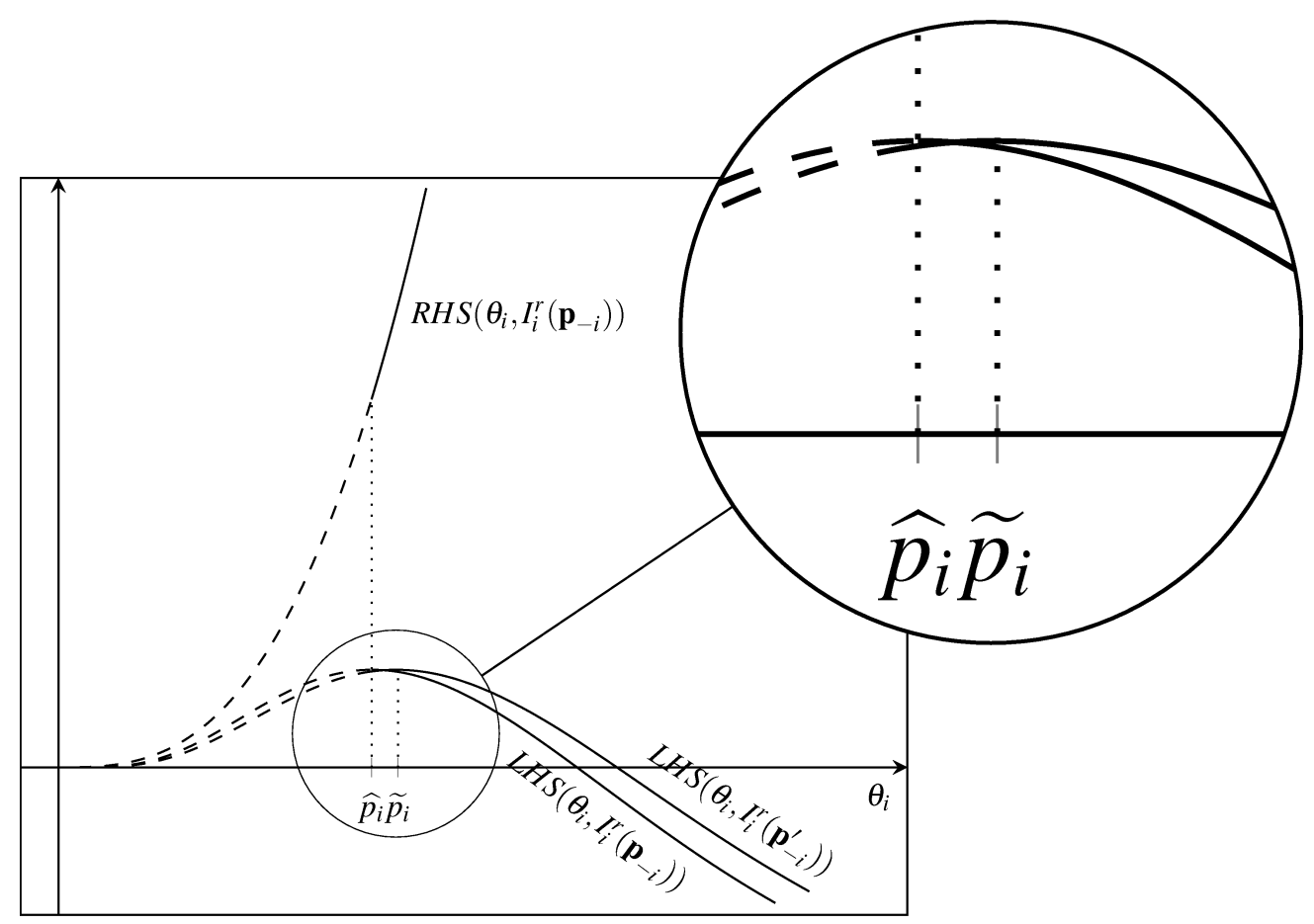

Figure 1: A situation like Case 1.(a): Say $\widehat{p}_{i}:=p_{i, \min }\left(\mathbf{p}_{-i}\right)=B R_{i}^{u}\left(\mathbf{p}_{-i}\right)$; $\widetilde{p}_{i}:=p_{i, \min }\left(\mathbf{p}_{-i}^{\prime}\right)$.

in $P_{i}\left(\mathbf{p}_{-i}\right)$, it results $\operatorname{RHS}\left(\theta_{i}, I_{i}^{r}\left(\mathbf{p}_{-i}^{\prime}\right)\right)<\operatorname{LHS}\left(\theta_{i}, I_{i}^{r}\left(\mathbf{p}_{-i}\right)\right)$. Because of the property of horizontal dilatations, it holds

$$
\operatorname{LHS}\left(\theta_{i}, I_{i}^{r}\left(\mathbf{p}_{-i}\right)\right)=\operatorname{LHS}\left(\frac{\theta_{i}}{c}, I_{i}^{r}\left(\mathbf{p}_{-i}^{\prime}\right)\right)
$$

and hence $\operatorname{RHS}\left(\theta_{i}, I_{i}^{r}\left(\mathbf{p}_{-i}^{\prime}\right)\right)<\operatorname{LHS}\left(\frac{\theta_{i}}{c}, I_{i}^{r}\left(\mathbf{p}_{-i}^{\prime}\right)\right)$. Being $\operatorname{LHS}\left(\theta_{i}, I_{i}^{r}\left(\mathbf{p}_{-i}^{\prime}\right)\right)$ strictly decreasing for $\theta_{i}>p_{i, \min }\left(\mathbf{p}_{-i}^{\prime}\right)$, then it results $\operatorname{LHS}\left(\frac{\theta_{i}}{c}, I_{i}^{r}\left(\mathbf{p}_{-i}^{\prime}\right)\right)$ $<\operatorname{LHS}\left(\theta_{i}, I_{i}^{r}\left(\mathbf{p}_{-i}^{\prime}\right)\right)$ and therefore it is also

$$
\operatorname{RHS}\left(\theta_{i}, I_{i}^{r}\left(\mathbf{p}_{-i}^{\prime}\right)\right)<\operatorname{LHS}\left(\theta_{i}, I_{i}^{r}\left(\mathbf{p}_{-i}^{\prime}\right)\right)
$$

in the whole interval $\theta_{i} \in\left[p_{i, \min }\left(\mathbf{p}_{-i}^{\prime}\right), p_{i, \max }\left(\mathbf{p}_{-i}\right)\right]$. As a consequence, the point of intersection of the graphs of $\operatorname{RHS}\left(\theta_{i}, I_{i}^{r}\left(\mathbf{p}_{-i}^{\prime}\right)\right)$ and $\operatorname{LHS}\left(\theta_{i}\right.$, $\left.I_{i}^{r}\left(\mathbf{p}_{-i}^{\prime}\right)\right)$ has abscissa $\tilde{\theta}$ greater than $p_{i, \max }\left(\mathbf{p}_{-i}\right)$. It involves that $B R_{i}^{u}\left(\mathbf{p}_{-i}^{\prime}\right)$ $>B R_{i}^{u}\left(\mathbf{p}_{-i}\right)$, whether or not $\tilde{\theta}$ is smaller than $p_{i, \max }\left(\mathbf{p}_{-i}^{\prime}\right)$, qed. 


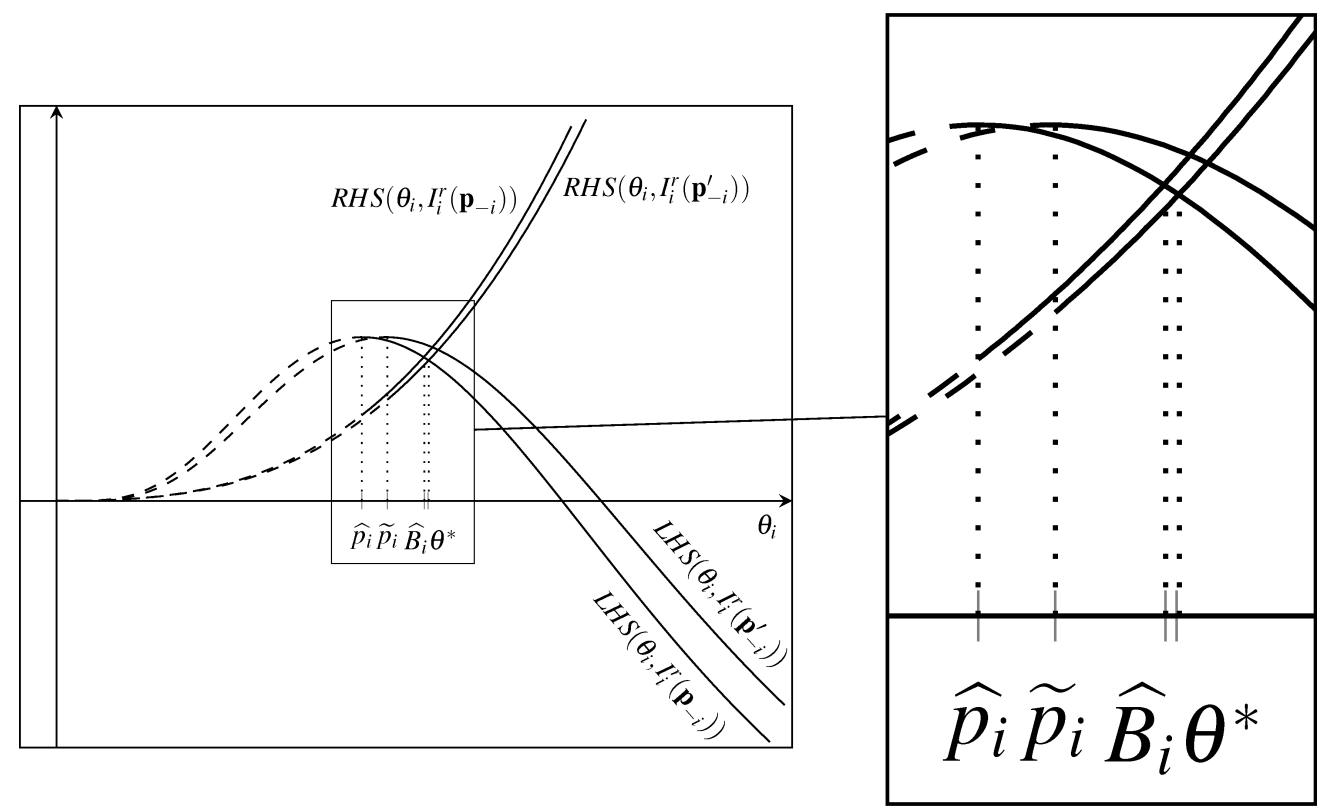

Figure 2: A situation like Case 1.(b).ii: Say $\widehat{p}_{i}:=p_{i, \min }\left(\mathbf{p}_{-i}\right) ; \quad \widetilde{p}_{i}:=$ $p_{i, \min }\left(\mathbf{p}_{-i}^{\prime}\right) ; \quad \widehat{B}_{i}=B R_{i}^{u}\left(\mathbf{p}_{-i}\right)$.

Monotonicity of the unconstrained Best Responses $B R_{i}^{u}$ has been therefore proven, for every $i \in \mathscr{N}$.

In the following, we are going to show the scalability property of the unconstrained Best Responses $B R_{i}^{u}\left(\mathbf{p}_{-i}\right)$, see Definition 9, for $p_{i} \geq p_{i, \min }\left(\mathbf{p}_{-i}\right)$.

Proof. [Scalability of the unconstrained Best Responses $B R_{i}^{u}\left(\mathbf{p}_{-i}\right)$.]

In what follows, we are going to show that for every $\alpha>1, \alpha \in \mathbb{R}$, and for every $i \in \mathscr{N}$ it results: $\alpha B R_{i}^{u}\left(\mathbf{p}_{-i}\right)>B R_{i}^{u}\left(\alpha \mathbf{p}_{-i}\right)$. Consider the power strategy $\alpha \mathbf{p}_{-i}$ of the other $N-1$ players. Consider all the possibilities that can occur for the Best Response $B R_{i}^{u}\left(\alpha \mathbf{p}_{-i}\right)$ of the $i$-th player to such a strategy together with the possibilities for the Best Response $B R_{i}^{u}\left(\mathbf{p}_{-i}\right)$. Four cases cover all the possible situations that can occur. We list them in the following.

1. Case $B R_{i}^{u}\left(\alpha \mathbf{p}_{-i}\right)=p_{i, \min }\left(\alpha \mathbf{p}_{-i}\right)$ and $B R_{i}^{u}\left(\mathbf{p}_{-i}\right)$ any in the strategy set $P_{i}\left(\mathbf{p}_{-i}\right)$ : it is $B R_{i}^{u}\left(\alpha \mathbf{p}_{-i}\right)=p_{i, \min }\left(\alpha \mathbf{p}_{-i}\right)=\frac{\gamma_{M}}{g_{i, i}} I_{i}^{r}\left(\alpha \mathbf{p}_{-i}\right)<\alpha \frac{\gamma_{M}}{g_{i, i}} I_{i}^{r}\left(\mathbf{p}_{-i}\right)=\alpha p_{i, \min }\left(\mathbf{p}_{-i}\right) \leq$ $\alpha B R_{i}^{u}\left(\mathbf{p}_{-i}\right)$ q.e.d., because of the definition of $p_{i, \min }$ and the scalability property of the Interference function, see Lemma 10. 
2. Case $B R_{i}^{u}\left(\alpha \mathbf{p}_{-i}\right)$ any in the strategy set $P_{i}\left(\alpha \mathbf{p}_{-i}\right)$ and $B R_{i}^{u}\left(\mathbf{p}_{-i}\right)=p_{i, \max }\left(\mathbf{p}_{-i}\right)$ : it is $B R_{i}^{u}\left(\alpha \mathbf{p}_{-i}\right) \leq p_{i, \max }\left(\alpha \mathbf{p}_{-i}\right)<\alpha p_{i, \max }\left(\mathbf{p}_{-i}\right)=\alpha B R_{i}^{u}\left(\mathbf{p}_{-i}\right)$, q.e.d., because of the scalability property of the Interference function.

3. Case $B R_{i}^{u}\left(\alpha \mathbf{p}_{-i}\right)$ abscissa of the (unique) point of intersection between the graphs of $R H S\left(\theta_{i}, I_{i}^{r}\left(\alpha_{\mathbf{p}_{-i}}\right)\right)$ and $\operatorname{LHS}\left(\theta_{i}, I_{i}^{r}\left(\alpha \mathbf{p}_{-i}\right)\right)$, and $B R_{i}^{u}\left(\mathbf{p}_{-i}\right)$ abscissa of the (unique) point of intersection between the graphs of $\operatorname{RHS}\left(\theta_{i}, I_{i}^{r}\left(\mathbf{p}_{-i}\right)\right)$ and $\operatorname{LHS}\left(\theta_{i}, I_{i}^{r}\left(\mathbf{p}_{-i}\right)\right)$, if they intersect, or $B R_{i}^{u}\left(\mathbf{p}_{-i}\right)=p_{i, \min }\left(\mathbf{p}_{-i}\right)$, if

$$
\operatorname{LHS}\left(\theta_{i}, I_{i}^{r}\left(\mathbf{p}_{-i}\right)\right)<\operatorname{RHS}\left(\theta_{i}, I_{i}^{r}\left(\mathbf{p}_{-i}\right)\right)
$$

for every $\theta_{i} \in P_{i}\left(\mathbf{p}_{-i}\right)$ (one of these situations is shown in Fig. 3). These assumptions imply that:

$$
\begin{aligned}
\operatorname{LHS}\left(B R_{i}^{u}\left(\alpha \mathbf{p}_{-i}\right), I_{i}^{r}\left(\alpha \mathbf{p}_{-i}\right)\right) & =R H S\left(B R_{i}^{u}\left(\alpha \mathbf{p}_{-i}\right), I_{i}^{r}\left(\alpha \mathbf{p}_{-i}\right)\right), \\
\operatorname{LHS}\left(B R_{i}^{u}\left(\mathbf{p}_{-i}\right), I_{i}^{r}\left(\mathbf{p}_{-i}\right)\right) & \leq \operatorname{RHS}\left(B R_{i}^{u}\left(\mathbf{p}_{-i}\right), I_{i}^{r}\left(\mathbf{p}_{-i}\right)\right) .
\end{aligned}
$$

The proof of scalability is difficult, because we cannot give an explicit expression for the (unconstrained) Best Responses. The first aspect to be considered is a consequence of the following chain of inequalities: if we put $k:=\frac{I_{i}^{r}\left(\mathbf{p}_{-i}\right)}{I_{i}^{i}\left(\alpha \mathbf{p}_{-i}\right)} \in(0,1)$, it results

$$
\alpha B R_{i}^{u}\left(\mathbf{p}_{-i}\right)>\frac{B R_{i}^{u}\left(\mathbf{p}_{-i}\right)}{k} \geq \frac{p_{i, \min }\left(\mathbf{p}_{-i}\right)}{k}=p_{i, \min }\left(\alpha \mathbf{p}_{-i}\right) .
$$

The first inequality is due to the scalability of the Interference function, that implies $\alpha k>1$. Therefore, from (27), it is certainly $\alpha B R_{i}^{u}\left(\mathbf{p}_{-i}\right)>p_{i, \min }\left(\alpha \mathbf{p}_{-i}\right)$. If it is $\alpha B R_{i}^{u}\left(\mathbf{p}_{-i}\right)>p_{i, \max }\left(\alpha \mathbf{p}_{-i}\right)$, the proof is ended, because $p_{i, \max }\left(\alpha \mathbf{p}_{-i}\right) \geq$ $B R_{i}^{u}\left(\alpha \mathbf{p}_{-i}\right)$ and therefore $\alpha B R_{i}^{u}\left(\mathbf{p}_{-i}\right)>B R_{i}^{u}\left(\alpha \mathbf{p}_{-i}\right)$. Otherwise, it is

$$
p_{i, \min }\left(\alpha \mathbf{p}_{-i}\right)<\alpha B R_{i}^{u}\left(\mathbf{p}_{-i}\right) \leq p_{i, \max }\left(\alpha \mathbf{p}_{-i}\right) .
$$

In this case, we need some more computations. The first step consists in showing that, for every $\alpha>1, \operatorname{LHS}\left(\alpha B R_{i}^{u}\left(\mathbf{p}_{-i}\right), I_{i}^{r}\left(\alpha \mathbf{p}_{-i}\right)\right)<\operatorname{LHS}\left(B R_{i}^{u}\left(\mathbf{p}_{-i}\right), I_{i}^{r}\left(\mathbf{p}_{-i}\right)\right)$. It is $\operatorname{LHS}\left(\theta_{i}, I_{i}^{r}\left(\alpha \mathbf{p}_{-i}\right)\right)=\operatorname{LHS}\left(k \theta_{i}, I_{i}^{r}\left(\mathbf{p}_{-i}\right)\right)$ with $k:=\frac{I_{i}^{r}\left(\mathbf{p}_{-i}\right)}{I_{i}^{r}\left(\alpha \mathbf{p}_{-i}\right)} \in(0,1)$, therefore $\operatorname{LHS}\left(\theta_{i}, I_{i}^{r}\left(\alpha \mathbf{p}_{-i}\right)\right)$ is a horizontal dilatation of $\operatorname{LHS}\left(\theta_{i}, I_{i}^{r}\left(\mathbf{p}_{-i}\right)\right)$ of factor $\frac{1}{k}>1$ and the following is true:

$$
\operatorname{LHS}\left(B R_{i}^{u}\left(\mathbf{p}_{-i}\right), I_{i}^{r}\left(\mathbf{p}_{-i}\right)\right)=\operatorname{LHS}\left(\frac{B R_{i}^{u}\left(\mathbf{p}_{-i}\right)}{k}, I_{i}^{r}\left(\alpha \mathbf{p}_{-i}\right)\right) .
$$

It results

$$
\operatorname{LHS}\left(\alpha B R_{i}^{u}\left(\mathbf{p}_{-i}\right), I_{i}^{r}\left(\alpha \mathbf{p}_{-i}\right)\right)<\operatorname{LHS}\left(\frac{B R_{i}^{u}\left(\mathbf{p}_{-i}\right)}{k}, I_{i}^{r}\left(\alpha \mathbf{p}_{-i}\right)\right)
$$


because $\operatorname{LHS}\left(\theta_{i}, I_{i}^{r}\left(\alpha \mathbf{p}_{-i}\right)\right)$ is decreasing for $\theta_{i}>p_{i, \min }\left(\alpha \mathbf{p}_{-i}\right)$ and $\alpha B R_{i}^{u}\left(\mathbf{p}_{-i}\right)>$ $\frac{B R_{i}^{u}\left(\mathbf{p}_{-i}\right)}{k} \geq \frac{p_{i, \min }\left(\mathbf{p}_{-i}\right)}{k}=p_{i, \min }\left(\alpha \mathbf{p}_{-i}\right)$. Eqs. (28) and (29) imply:

$$
\operatorname{LHS}\left(\alpha B R_{i}^{u}\left(\mathbf{p}_{-i}\right), I_{i}^{r}\left(\alpha \mathbf{p}_{-i}\right)\right)<\operatorname{LHS}\left(B R_{i}^{u}\left(\mathbf{p}_{-i}\right), I_{i}^{r}\left(\mathbf{p}_{-i}\right)\right)
$$
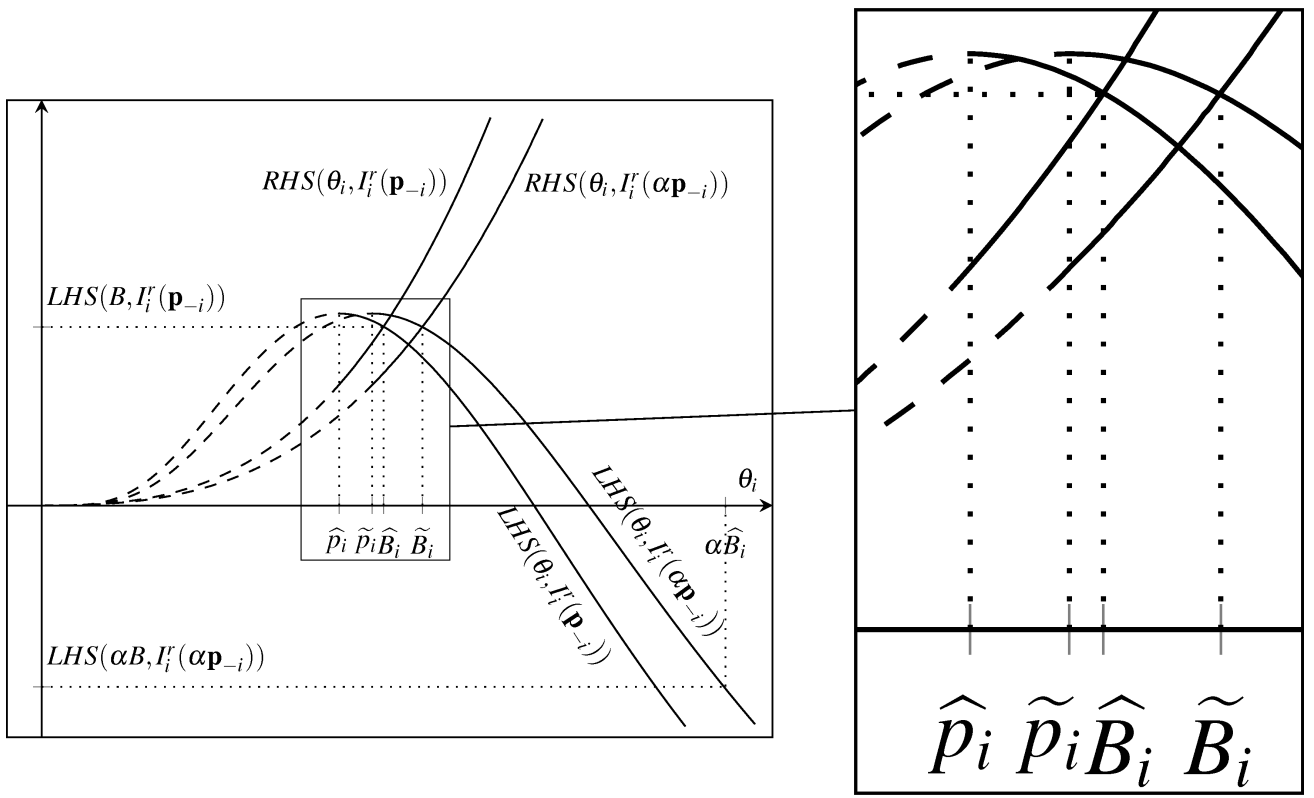

Figure 3: A possible situation in Case 3: Say $\widehat{p}_{i}:=p_{i, \min }\left(\mathbf{p}_{-i}\right) ; \quad \widetilde{p}_{i}:=$ $p_{i, \min }\left(\alpha \mathbf{p}_{-i}\right) ; \quad \widehat{B}_{i}=B R_{i}^{u}\left(\mathbf{p}_{-i}\right) ; \quad \widetilde{B}_{i}=B R_{i}^{u}\left(\alpha \mathbf{p}_{-i}\right)$.

The second step consists in showing that, for every $\alpha>1$,

$$
R H S\left(B R_{i}^{u}\left(\mathbf{p}_{-i}\right), I_{i}^{r}\left(\mathbf{p}_{-i}\right)\right)<\frac{1}{\alpha^{2}} R H S\left(\alpha B R_{i}^{u}\left(\mathbf{p}_{-i}\right), I_{i}^{r}\left(\alpha \mathbf{p}_{-i}\right)\right)
$$


By rearranging Eq. (19), with $p_{i}$ substituted by $B R_{i}^{u}\left(\mathbf{p}_{-i}\right)$, we obtain:

$$
\begin{aligned}
& \operatorname{RHS}\left(B R_{i}^{u}\left(\mathbf{p}_{-i}\right), I_{i}^{r}\left(\mathbf{p}_{-i}\right)\right) \\
& =\frac{\alpha^{2}\left[B R_{i}^{u}\left(\mathbf{p}_{-i}\right)\right]^{2}}{\alpha^{2} W R_{i}}\left[\beta-\delta \exp \left(-\mu \frac{\alpha B R_{i}^{u}\left(\mathbf{p}_{-i}\right) \sum_{k=1, k \neq i}^{N} g_{i, k}}{\alpha I_{i}^{r}\left(\mathbf{p}_{-i}\right)}\right)\right. \\
& \left.+\delta \mu \frac{\sum_{k=1, k \neq i}^{N} g_{i, k}}{\alpha I_{i}^{r}\left(\mathbf{p}_{-i}\right)} \exp \left(-\mu \frac{\alpha B R_{i}^{u}\left(\mathbf{p}_{-i}\right) \sum_{k=1, k \neq i}^{N} g_{i, k}}{\alpha I_{i}^{r}\left(\mathbf{p}_{-i}\right)}\right) \alpha B R_{i}^{u}\left(\mathbf{p}_{-i}\right)\right]
\end{aligned}
$$

Provided that

$$
\begin{aligned}
& \frac{1}{\alpha^{2}} \operatorname{RHS}\left(\alpha B R_{i}^{u}\left(\mathbf{p}_{-i}\right), I_{i}^{r}\left(\alpha \mathbf{p}_{-i}\right)\right) \\
& =\frac{\left[\alpha B R_{i}^{u}\left(\mathbf{p}_{-i}\right)\right]^{2}}{\alpha^{2} W R_{i}}\left[\beta-\delta \exp \left(\begin{array}{c}
\alpha B R_{i}^{u}\left(\mathbf{p}_{-i}\right) \sum_{k=1, k \neq i}^{N} g_{i, k} \\
I_{i}^{r}\left(\alpha \mathbf{p}_{-i}\right)
\end{array}\right)\right. \\
& +\delta \mu \frac{\sum_{k=1, k \neq i}^{N} g_{i, k}}{I_{i}^{r}\left(\alpha \mathbf{p}_{-i}\right)} \exp \left(-\mu \frac{\alpha B R_{i}^{u}\left(\mathbf{p}_{-i}\right) \sum_{k=1, k \neq i}^{N} g_{i, k}}{I_{i}^{r}\left(\alpha \mathbf{p}_{-i}\right)} \mid \alpha B R_{i}^{u}\left(\mathbf{p}_{-i}\right)\right],
\end{aligned}
$$

inequality (31) holds if and only if the following holds for every $\alpha>1$ :

$$
\begin{aligned}
& \left(\mu \frac{\sum_{k=1, k \neq i}^{N} g_{i, k}}{\alpha I_{i}^{r}\left(\mathbf{p}_{-i}\right)} \alpha B R_{i}^{u}\left(\mathbf{p}_{-i}\right)-1\right) \exp \left(\begin{array}{c}
\alpha B R_{i}^{u}\left(\mathbf{p}_{-i}\right) \sum_{k=1, k \neq i}^{N} g_{i, k} \\
\left.-\mu \frac{\alpha I_{i}^{r}\left(\mathbf{p}_{-i}\right)}{2}\right)
\end{array}\right. \\
& <\left(\mu \frac{\sum_{k=1, k \neq i}^{N} g_{i, k}}{I_{i}^{r}\left(\alpha \mathbf{p}_{-i}\right)} \alpha B R_{i}^{u}\left(\mathbf{p}_{-i}\right)-1\right) \exp \left(-\mu \frac{\alpha B R_{i}^{u}\left(\mathbf{p}_{-i}\right) \sum_{k=1, k \neq i}^{N} g_{i, k}}{I_{i}^{r}\left(\alpha \mathbf{p}_{-i}\right)}\right) .
\end{aligned}
$$

Consider a new variable $z_{i}:=\mu \alpha B R_{i}^{u}\left(\mathbf{p}_{-i}\right) \sum_{k=1, k \neq i}^{N} g_{i, k}$. We recall that we are 
assuming $p_{i, \min }\left(\alpha \mathbf{p}_{-i}\right)<\alpha B R_{i}^{u}\left(\mathbf{p}_{-i}\right) \leq p_{i, \max }\left(\alpha \mathbf{p}_{-i}\right)$, therefore it is

$$
z_{i} \in\left(\frac{\gamma_{M}}{g_{i, i}} \mu I_{i}^{r}\left(\alpha \mathbf{p}_{-i}\right) \sum_{k=1, k \neq i}^{N} g_{i, k}, 2 I_{i}^{r}\left(\alpha \mathbf{p}_{-i}\right)\right]
$$

The previous inequality holds if and only if for every $\alpha>1$ it results:

$$
\begin{aligned}
{\left[\frac{z_{i}}{\alpha I_{i}^{r}\left(\mathbf{p}_{-i}\right)}-1\right] } & \exp \left(-\frac{z_{i}}{\alpha I_{i}^{r}\left(\mathbf{p}_{-i}\right)}\right) \\
& <\left[\frac{z_{i}}{I_{i}^{r}\left(\alpha \mathbf{p}_{-i}\right)}-1\right] \exp \left(-\frac{z_{i}}{I_{i}^{r}\left(\alpha \mathbf{p}_{-i}\right)}\right)
\end{aligned}
$$

Say $h\left(z_{i}, a\right):=\left[\frac{z_{i}}{a}-1\right] \exp \left(-\frac{z_{i}}{a}\right)$, with $a>0$. The study of such a family of curves, for non-negative values of $z_{i}$, let us state that the graph of $h\left(z_{i}, a\right)$ intercepts the $y$ axis in the point with coordinates $(0,-1)$, increases for $z_{i}$ increasing, intercepts the $z_{i}$-axis in the point with coordinates $(a, 0)$, still increases until it reaches the point with coordinates $\left(2 a, \mathrm{e}^{-2}\right)$ and then decreases tending to zero when $z_{i}$ tends to infinity. If $a>a^{\prime}$, it results $h\left(z_{i}, a\right)<h\left(z_{i}, a^{\prime}\right)$ certainly if $z_{i} \in\left(0,2 a^{\prime}\right]$. Say $a:=\alpha I_{i}^{r}\left(\mathbf{p}_{-i}\right)$ and $a^{\prime}:=I_{i}^{r}\left(\alpha \mathbf{p}_{-i}\right)$. It is $a>a^{\prime}$, and therefore $h\left(z_{i}, \alpha I_{i}^{r}\left(\mathbf{p}_{-i}\right)\right)<h\left(z_{i}, I_{i}^{r}\left(\alpha \mathbf{p}_{-i}\right)\right)$ certainly if $z_{i} \in\left(0,2 I_{i}^{r}\left(\alpha \mathbf{p}_{-i}\right)\right]$. In our case, inequality (32) is therefore satisfied and hence (31) is verified for every $\alpha>1$.

By considering (30), (26) and (31), we can state that:

$$
\operatorname{LHS}\left(\alpha B R_{i}^{u}\left(\mathbf{p}_{-i}\right), I_{i}^{r}\left(\alpha \mathbf{p}_{-i}\right)\right)<\frac{1}{\alpha^{2}} \operatorname{RHS}\left(\alpha B R_{i}^{u}\left(\mathbf{p}_{-i}\right), I_{i}^{r}\left(\alpha \mathbf{p}_{-i}\right)\right) .
$$

Consider a new variable for the previous inequality, say $Y_{i}:=\alpha B R_{i}^{u}\left(\mathbf{p}_{-i}\right)$. Therefore we can conclude that the two variables $B R_{i}^{u}\left(\alpha \mathbf{p}_{-i}\right)$ and $Y_{i}$ satisfy Eq. (34) and inequality (35) respectively:

$$
\begin{gathered}
\operatorname{LHS}\left(B R_{i}^{u}\left(\alpha \mathbf{p}_{-i}\right), I_{i}^{r}\left(\alpha \mathbf{p}_{-i}\right)\right)=\operatorname{RHS}\left(B R_{i}^{u}\left(\alpha \mathbf{p}_{-i}\right), I_{i}^{r}\left(\alpha \mathbf{p}_{-i}\right)\right), \\
\operatorname{LHS}\left(Y_{i}, I_{i}^{r}\left(\alpha \mathbf{p}_{-i}\right)\right)<\frac{1}{\alpha^{2}} \operatorname{RHS}\left(Y_{i}, I_{i}^{r}\left(\alpha \mathbf{p}_{-i}\right)\right) .
\end{gathered}
$$

It means that $\theta_{i}=B R_{i}^{u}\left(\alpha \mathbf{p}_{-i}\right)$ is the (unique) solution of equation

$$
\operatorname{LHS}\left(\theta_{i}, I_{i}^{r}\left(\alpha \mathbf{p}_{-i}\right)\right)=\operatorname{RHS}\left(\theta_{i}, I_{i}^{r}\left(\alpha \mathbf{p}_{-i}\right)\right)
$$

and $\theta_{i}=Y_{i}$ satisfies inequality:

$$
\operatorname{LHS}\left(\theta_{i}, I_{i}^{r}\left(\alpha \mathbf{p}_{-i}\right)\right)<\frac{1}{\alpha^{2}} R H S\left(\theta_{i}, I_{i}^{r}\left(\alpha \mathbf{p}_{-i}\right)\right) .
$$




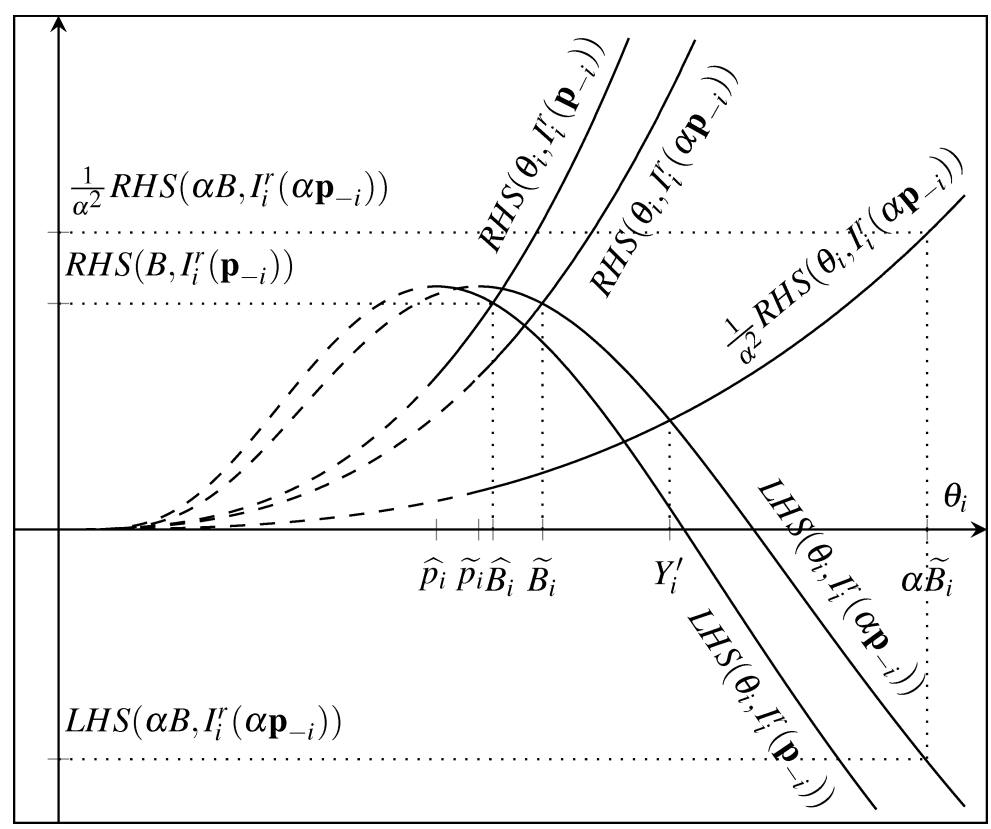

Figure 4: A possible situation in Case 3: Say $\widehat{p}_{i}:=p_{i, \min }\left(\mathbf{p}_{-i}\right) ; \quad \widetilde{p}_{i}:=$ $p_{i, \min }\left(\alpha \mathbf{p}_{-i}\right) ; \quad \widehat{B}_{i}=B R_{i}^{u}\left(\mathbf{p}_{-i}\right) ; \quad \widetilde{B}_{i}=B R_{i}^{u}\left(\alpha \mathbf{p}_{-i}\right)$.

Let $Y_{i}^{\prime}$ be the solution of equation:

$$
\operatorname{LHS}\left(\theta_{i}, I_{i}^{r}\left(\alpha \mathbf{p}_{-i}\right)\right)=\frac{1}{\alpha^{2}} \operatorname{RHS}\left(\theta_{i}, I_{i}^{r}\left(\alpha \mathbf{p}_{-i}\right)\right) .
$$

The graphs of $\operatorname{LHS}\left(\theta_{i}, I_{i}^{r}\left(\alpha \mathbf{p}_{-i}\right)\right)$ and $\operatorname{RHS}\left(\theta_{i}, I_{i}^{r}\left(\alpha \mathbf{p}_{-i}\right)\right)$ with respect to variable $\theta_{i}$ intersect in a point whose abscissa is $\theta_{i}=B R_{i}^{u}\left(\alpha \mathbf{p}_{-i}\right)$; the graphs of functions $\operatorname{LHS}\left(\theta_{i}, I_{i}^{r}\left(\alpha \mathbf{p}_{-i}\right)\right)$ and $\frac{1}{\alpha^{2}} \operatorname{RHS}\left(\theta_{i}, I_{i}^{r}\left(\alpha \mathbf{p}_{-i}\right)\right)$ intersect in a point whose abscissa is $\theta_{i}=Y_{i}^{\prime}$, see Fig. 4. By comparing Eqs. (36) and (38), one evinces that their left hand sides are the same, therefore they have the same graphs, while the right hand side of (38) is the same of the right hand side of (36) divided by $\alpha^{2}$, with $\alpha>1$, therefore the graph of $\frac{1}{\alpha^{2}} R H S\left(\theta_{i}, I_{i}^{r}\left(\alpha \mathbf{p}_{-i}\right)\right)$ is placed below the one of $\operatorname{RHS}\left(\theta_{i}, I_{i}^{r}\left(\alpha \mathbf{p}_{-i}\right)\right)$. This implies that the abscissa $\theta_{i}=Y_{i}^{\prime}$ of the point of intersection between the graphs of $\operatorname{LHS}\left(\theta_{i}, I_{i}^{r}\left(\alpha \mathbf{p}_{-i}\right)\right)$ and $\frac{1}{\alpha^{2}} \operatorname{RHS}\left(\theta_{i}, I_{i}^{r}\left(\alpha \mathbf{p}_{-i}\right)\right)$ is placed on the right of the abscissa $\theta_{i}=B R_{i}^{u}\left(\alpha \mathbf{p}_{-i}\right)$ of the point of intersection between the graphs of $\operatorname{LHS}\left(\theta_{i}, I_{i}^{r}\left(\alpha_{\mathbf{p}_{-i}}\right)\right)$ and $\operatorname{RHS}\left(\theta_{i}, I_{i}^{r}\left(\alpha \mathbf{p}_{-i}\right)\right)$, hence $Y_{i}^{\prime}>B R_{i}^{u}\left(\alpha \mathbf{p}_{-i}\right)$ for every $\alpha>1$. To conclude we just have to observe that all $Y_{i}$ solving inequality (37) are such that $Y_{i}>Y_{i}^{\prime}$, therefore $Y_{i}>Y_{i}^{\prime}>B R_{i}^{u}\left(\alpha \mathbf{p}_{-i}\right)$ for every $\alpha>1$. By substituting $Y_{i}=\alpha B R_{i}^{u}\left(\mathbf{p}_{-i}\right)$, one eventually obtains $\alpha B R_{i}^{u}\left(\mathbf{p}_{-i}\right)>$ 
$B R_{i}^{u}\left(\alpha \mathbf{p}_{-i}\right)$ for every $\alpha>1$, q.e.d.

4. Case $B R_{i}^{u}\left(\alpha \mathbf{p}_{-i}\right)=p_{i, \max }\left(\alpha \mathbf{p}_{-i}\right)$ (in case $\operatorname{LHS}\left(\theta_{i}, I_{i}^{r}\left(\alpha \mathbf{p}_{-i}\right)\right)>\operatorname{RHS}\left(\theta_{i}, I_{i}^{r}\left(\alpha \mathbf{p}_{-i}\right)\right)$ for every $\theta_{i} \in P\left(\alpha \mathbf{p}_{-i}\right)$ ), and $B R_{i}^{u}\left(\mathbf{p}_{-i}\right)$ like in the previous case. It still holds Eq. (33) and therefore the following chain holds:

$$
\begin{aligned}
\operatorname{LHS}\left(\alpha B R_{i}^{u}\left(\mathbf{p}_{-i}\right), I_{i}^{r}\left(\alpha \mathbf{p}_{-i}\right)\right)<\frac{1}{\alpha^{2}} \operatorname{RHS}\left(\alpha B R_{i}^{u}\left(\mathbf{p}_{-i}\right), I_{i}^{r}\left(\alpha \mathbf{p}_{-i}\right)\right) \\
\\
<\operatorname{RHS}\left(\alpha B R_{i}^{u}\left(\mathbf{p}_{-i}\right), I_{i}^{r}\left(\alpha \mathbf{p}_{-i}\right)\right) .
\end{aligned}
$$

We conclude that $\alpha B R_{i}^{u}\left(\mathbf{p}_{-i}\right) \notin P\left(\alpha \mathbf{p}_{-i}\right)$ and, as a logical consequence, $\alpha B R_{i}^{u}\left(\mathbf{p}_{-i}\right)$ $>p_{i, \max }\left(\alpha \mathbf{p}_{-i}\right)=B R_{i}^{u}\left(\alpha \mathbf{p}_{-i}\right)$, q.e.d.

We have therefore proven that the unconstrained Best Responses have the scalability property.

We have proven that the unconstrained Best Responses are standard functions, in the temporary assumption that there is not a maximum power constraint $P_{i, \max }$. The last step in the proof is the inclusion of the maximum power constraint $P_{i, \max }$ back. The Best Response $B R_{i}\left(\mathbf{p}_{-i}\right)$ of the constrained problem is simply:

$$
\begin{aligned}
B R_{i}\left(\mathbf{p}_{-i}\right) & := \begin{cases}B R_{i}^{u}\left(\mathbf{p}_{-i}\right) & \text { if } B R_{i}^{u}\left(\mathbf{p}_{-i}\right) \leq P_{i, \max } \\
P_{i, \max } & \text { if } B R_{i}^{u}\left(\mathbf{p}_{-i}\right)>P_{i, \max }\end{cases} \\
& =\min \left\{B R_{i}^{u}\left(\mathbf{p}_{-i}\right), P_{i, \max }\right\} .
\end{aligned}
$$

We have proven that functions $B R_{i}^{u}$ are standard functions and also the constant function which assigns the constant value $P_{i, \max }$ to every power vector is trivially standard, see [48]. Hence function $B R_{i}$, see Eq. (39), is the minimum between two standard functions and it is therefore standard, see [36] and [48].

Theorem 12. The GNEP defined in Section 3 has a unique GNE that can be reached by the Best Response Algorithm.

Proof. Indeed, the Best Response $B R(\mathbf{p})=\left(B R_{i}\left(\mathbf{p}_{-i}\right)\right)_{i \in \mathscr{N}}$ (where $B R_{i}$ are singlevalued) is a standard function, see Lemma 11, and the existence of a GNE has been proven in Theroem 8; it is enough to state that the GNE is unique and that it can be reached by the Best Response Algorithm, see [36], [37] and [46]. 


\section{Conclusions}

We have presented a Generalized Nash Equilibrium Problem to model the coexistence of primary and secondary users in the same frequency band in a Cognitive Radio scenario for Dynamic Spectrum Access (DSA). Assuming that the secondary utilities with pricing depend on the simultaneous choices of players ([28]), we have developed a theoretic analysis showing existence and uniqueness of the Generalized Nash Equilibrium and convergence of the Best Response Algorithm to it. The given conditions just impose a lower level as given in [25] and an upper level to the SINR of the radio devices, based on the sensitivity of the set of users to interference as a parameter of the game. Such conditions are easy to be satisfied by the users, as they do not need to know every single power allocated by all the other users, generally not available, but they just avail local information, i.e. the total interference they experience near their own receiver.

\section{References}

[1] Committee on Scientific Use of the Radio Spectrum; Committee on Radio Frequencies; Board on Physics and Astronomy; Division on Engineering and Physical Sciences; National Research Council, Spectrum Management for Science in the 21st Century, The National Academies Press, Washington, D.C. (2010).

[2] Federal Communications Commission (FCC) - Spectrum policy task force, Report, ET Docket No. 02-135 (November 2002) [online]. Available from: https: //apps.fcc.gov/ edocs_public/ attachmatch/ DOC-228542A1. pdf [Accessed July 2, 2014].

[3] F.K. Jondral, From Maxwell's Equations to Cognitive Radio, Proceedings of the 3rd International Conference on Cognitive Radio Oriented Wireless Networks and Communications, CrownCom 2008, Singapore (15-17 May 2008). doi: 10.1109/CROWNCOM.2008.4562458.

[4] Radio Spectrum Policy Group (RSPG), RSPG Opinion on the Radio Spectrum Policy Programme, RSPG10-330 Final (June 2010) [online]. Available from: http://rspg.ec.europa.eu/_documents/documents/opinions/rspg10_ 330_rspp_opinion.pdf [Accessed July 2, 2014].

[5] Radio Spectrum Policy Group (RSPG), RSPG Opinion on Cognitive Technologies, RSPG10-348 Final (February 2011) [online]. Available from: http://rspg.ec.europa.eu/_documents/documents/meeting/rspg_10_ 348_ct_opinion_final.pdf [Accessed July 2, 2014]. 
[6] Radio Spectrum Policy Group (RSPG), Report on Collective use of Spectrum (CUS) and other spectrum sharing approaches, RSPG11-392 Final (November 2011) [online]. Available from: http://rspg.ec.europa.eu/_documents/documents/meeting/rspg26/ rspg11_392_report_CUS_other_approaches_final.pdf [Accessed July 2, 2014].

[7] Radio Spectrum Policy Group (RSPG), RSPG Opinion on Best practices regarding the use of spectrum by public sectors, RSPG09-258 (February 2009) [online]. Available from: http://rspg.ec.europa.eu/_documents/documents/opinions/rspg09_ 258_rspgopinion_pus_final.pdf [Accessed July 2, 2014].

[8] G. Li, G.L. Stüber, Orthogonal Frequency Division Multiplexing for Wireless Communications, Springer, New York (2006). doi: 10.1007/0-387-30235-2.

[9] F. Tamburini, E. Mari, A. Sponselli, B. Thidé, A. Bianchini, F. Romanato, Encoding many channels on the same frequency through radio vorticity: first experimental test, New Journal of Physics, 14 No. 033001 (2012), 1-17. doi: 10.1088/1367-2630/14/3/033001.

[10] R. Tandra, S.M. Mishra, A. Sahai, What is a spectrum hole and what does it take to recognize one?, Proc. IEEE, 97, No. 5 (2009), 824-848. doi: 10.1109/JPROC.2009.2015710.

[11] I.F. Akyildiz, W.Y. Lee, M.C. Vuran, S. Mohanty, NeXt generation/dynamic spectrum access/cognitive radio wireless networks: A survey, Computer Networks, 50 (2006), 2127-59. doi: 10.1016/j.comnet.2006.05.001 .

[12] Federal Communications Commission (FCC), Notice of proposed rulemaking and order, ET Docket No. 03-322 (December 2003) [online]. Available from: https: //apps.fcc.gov/ edocs_public/ attachmatch/FCC-03-322A1. pdf [Accessed July 2, 2014].

[13] S. Haykin, D.J. Thomson, J.H. Reed, Spectrum sensing for cognitive radio, Proceedings of the IEEE, 97, No. 5 (2009), 849-877. doi: 10.1109/JPROC.2009.2015711.

[14] S. Haykin, Cognitive radio: Brain-empowered wireless communications, IEEE J Select. Areas Commun., 23, No. 2 (2005), 201-220. doi: 10.1109/JSAC.2004.839380. 
[15] F.K. Jondral, Software-Defined Radio: Basics and Evolution to Cognitive Radio, EURASIP J. on Wireless Comm. and Networking, 3 (2005), 275-283. doi: 10.1155/WCN.2005.275.

[16] J.III Mitola, G.Q.Jr Maguire, Cognitive radio: making software radios more personal, IEEE Personal Communications, 6, No. 4 (1999), 13-18. doi: 10.1109/98.788210.

[17] J.III Mitola, Cognitive radio: An integrated agent architecture for software defined radio, Dissertation for Doctor of Technology, Royal Inst. Technol. (KTH), ISSN 1403-5286, ISRN KTH/IT/AVH 00/01,-SE, Stockholm, Sweden (2000).

[18] A. Zappone, Z. Chong, E.A. Jorswieck, S. Buzzi, Energy-Aware Competitive Power Control in Relay-Assisted Interference Wireless Networks, IEEE Transactions on Wireless Communications, 12, No. 4 (2013), 1860-71. doi: 10.1109/TWC.2013.031313.121103.

[19] B. Wang, Y. Wu, K.J.R. Liu, Game Theory for Cognitive Radio Networks: an Overview, Computer Networks, 54, No. 14 (2010), 2537-61. doi: 10.1016/j.comnet.2010.04.004.

[20] D. Famolari, N. Mandayam, D. Goodman, V. Shah, A new framework for power control in wireless data networks: games, utility, and pricing, In: Wireless Multimedia Network Technologies, eds. Ganesh, Pahlavan and Zvonar, Kluwer Academic (1999), 289-310. doi: 10.1007/0-306-47330-5_16.

[21] N. Feng, N.B. Mandayam, D.J. Goodman, Joint power and rate optimization for wireless data services based on utility functions, Proc. CISS, 1 (1999), 109-113.

[22] D.J. Goodman, N.B. Mandayam, Power control for wireless data, IEEE Pers. Comm. Mag., 7 (2000), 45-54. doi: 10.1109/98.839331.

[23] H. Ji, D. Huang, Non-cooperative uplink power control in cellular radio systems, Journal Wireless Networks - Special issue transmitter power control, 4, No. 3 (1998), 233-240.

[24] V. Shah, N. Mandayam, D. Goodman, Power Control for Wireless Data based on Utility and Pricing, Proc. PIMRC '98 (1998), 1427-32. doi: 10.1109/PIMRC.1998.731433.

[25] C.U. Saraydar, N. Mandayam, D. Goodman, Efficient power control via pricing in wireless data networks, IEEE Trans. Comm., 50 (2002), 291-303. doi: $10.1109 / 26.983324$. 
[26] C.K. Tan, M.L. Sim, T.C. Chuah, Fair power control for wireless ad hoc networks using game theory with pricing scheme, IET Commun., 4, No. 3 (2010), 322-333. doi: 10.1049/iet-com.2009.0225.

[27] E. Del Re, R. Pucci, L.S. Ronga, Energy Efficient Resource Allocation Game for Cognitive Radio, CogART '11: Proceedings of the 4th International Conference on Cognitive Radio and Advanced Spectrum Management, Barcelona, Spain (2629 October 2011). doi: 10.1145/2093256.2093313.

[28] L. Prati, A Generalized Nash Equilibrium Problem for Centralized Dynamic Spectrum Leasing, Int. J. Pure Appl. Math., 85, No. 2 (2013), 367-382. doi: 10.12732/ijpam.v85i2.13.

[29] G. Debreu, A social equilibrium existence theorem, Proceedings of the National Academy of Sciences of the USA, 38 (1952), 886-893. doi: 10.1073/pnas.38.10.886.

[30] N. Feng, S.C. Mau, N.B. Mandayam, Pricing and power control for joint network-centric and user-centric radio resource management, IEEE Trans. Comm., 52 (2004), 1547-57. doi: 10.1109/TCOMM.2004.833191.

[31] K.J. Arrow, G. Debreu, Existence of an equilibrium for a competitive economy, Econometrica, 22 (1954), 265-290. doi: 10.2307/1907353.

[32] F. Facchinei, C. Kanzow, Generalized Nash Equilibrium Problems, Ann. Oper. Res., 175 (2010), 177-211. doi: 10.1007/s10479-009-0653-х .

[33] J.F. Nash, Equilibrium Points in n-Person Games, Proc. Nat. Acad. Sci. U.S.A., 36 (1950), 48-49. doi: 10.1073/pnas.36.1.48.

[34] J.F. Nash, Non-cooperative Games, Ann. of Math., 54 (1951), 286-295. doi: $10.2307 / 1969529$.

[35] D. Fudenberg, J. Tirole, Game Theory, MIT Press, Cambridge (1991).

[36] R.D. Yates, A framework for uplink power control in cellular radio systems, IEEE J. Select. Areas Commun., 13 (1995), 1341-47. doi: 10.1109/49.414651

[37] E. Altman, Z. Altman, S-modular games and power control in wireless networks, IEEE Transactions on Automatic Control, 48, No. 5 (2003), 839-842. doi: 10.1109/TAC.2003.811264. 
[38] D.M. Topkis, Minimizing a submodular function on a lattice, Operations research, 26 (1978), 305-321. doi: 10.1287/opre.26.2.305.

[39] D.M. Topkis, Equilibrium points in nonzero-sum n-person submodular games, SIAM J. Control Optim., 17 (1979), 773-778. doi: 10.1137/0317054.

[40] D.M. Topkis, Supermodularity and Complementarity, Princeton University Press, Princeton (1998).

[41] P. Milgrom, J. Roberts, Rationalizability, Learning, and Equilibrium in Games with Strategic Complementarities, Econometrica, 58, No. 6 (1990), 1255-77. doi: $10.2307 / 2938316$.

[42] P. Milgrom, C. Shannon, Monotone Comparative Statics, Econometrica, 62 (1994), 157-180. doi: 10.2307/2951479.

[43] X. Vives, Complementarities and games: new developments, Journal of Economic Literature, 43 (2005), 437-79. doi: 10.1257/0022051054661558.

[44] T. Ichiishi, Game Theory for Economic Analysis, Academic Press, New York (1983).

[45] K.K. Leung, C.W. Sung, W.S. Wong, T.M. Lok, Convergence Theorem for a General Class of Power-Control Algorithms, IEEE Transactions on Communications, 52, No. 9 (2004), 1566-74. doi: 10.1109/TCOMM.2004.833140.

[46] G. Bacci, M. Luise, A Game-Theoretic Perspective on Code Synchronization for CDMA Wireless Systems, IEEE Journal on Selected Areas in Communications, 30, No. 1 (2012), 107-118. doi: 10.1109/JSAC.2012.120110.

[47] G. Bacci, L. Sanguinetti, M. Luise, H.V. Poor, A Game-Theoretic Approach for Energy-Efficient Contention-Based Synchronization in OFDMA Systems, IEEE Transactions on Signal Processing, 61, No. 5 (2013), 1258-71. doi: 10.1109/TSP.2012.2231679.

[48] R.D. Yates, S. Gupta, C. Rose, S. Sohn, Soft dropping power control, Proceedings of The IEEE Vehicular Technology Conference VTC 97 (1997), 1694-98. doi: 10.1109/VETEC.1997.605847. 
